\title{
Parametric study on the optimal tuning of an inertial actuator for vibration control of a plate: theory and experiments
}

\author{
Stefano Camperi*, Maryam Ghandchi Tehrani, Stephen John Elliott \\ Institute of Sound and Vibration Research, Highfield, Southampton SO171BJ, UK
}

\begin{abstract}
This paper presents a theoretical and experimental tuning of the velocity feedback gain of a plate with an inertial actuator. The objective of the study is to analyse a direct velocity feedback control unit, which can be tuned locally and yet providing a global vibration reduction. This is achieved through the knowledge of the velocity signal and the actuator dynamics, without information on the plate dynamics. In practice, an electrical input is provided to the actuator, proportional to the local velocity of the structure, in such a way to generate active damping. The tuning is performed by maximising the power absorbed by the inertial actuator from the structure, and this is shown to be equivalent to the minimisation of the global level of vibration, estimated through the kinetic energy of the structure. Nine accelerometers have been used, and the performance for several values of different feedback gains has been investigated. Moreover, the influence of the frequency range of integration in the tuning of the velocity feedback gain is considered, and it is found experimentally that a broadband reduction up to $5 \mathrm{~dB}$ can be achieved, as expected from the numerical model. The absorbed power from the plate by the control unit is found to be negative below the first natural frequency of the inertial actuator, when the feedback control is implemented. This is due to the fact that, although a collocated velocity feedback is implemented, the control system is only conditionally stable because of the actuator dynamics. The performance of active control is found to reduce dramatically, if instability occurs for gains lower than the optimal one. For this reason, the work is enriched with a parametric study on the plate-actuator pair, in which the influence of the dynamic properties of the plate and the inertial actuator are investigated. The effectiveness of the active control is found to depend on the mass ratio between the actuator and the plate. In particular, for low mass ratios, the system well approximate the ideal case, in which a control force is proportional to the velocity of the plate, but for high mass ratios, a small amount of active damping can be introduced.
\end{abstract}

Keywords:

active velocity feedback, optimal tuning, power absorption, inertial actuator, structural vibration

\section{Introduction}

This paper presents a comprehensive study on tuning of the feedback gain of an inertial actuator for vibration control of a plate, through the minimisation of the total vibration of the structure and the maximisation of the absorbed power by the control unit. This work is an extension of the study presented in Ref. [1] to the case where an inertial actuator is considered. In particular, the aim of this work is to investigate the design of a self-contained device for active damping, which can be tuned to minimise the global level of vibration of a plate through the velocity feedback signal and a knowledge of the actuator dynamics, without information on the plate dynamics. The tuning process, as well as the amount of reduction achievable, is found to be related to the dynamic coupling between the actuator and the plate. For this reason, the optimisation of the feedback gain is investigated with a parametric study. Moreover, the results are validated with some experiments.

\footnotetext{
${ }^{*}$ Corresponding author

Email address: s. camperi@soton.ac.uk (Stefano Camperi)
} 
The flexural dynamic behaviour of lightly damped structures subjected to broadband random excitation, at low frequencies, is mostly determined by their resonances, which can be effectively reduced with active damping. This can be achieved with several control strategies based on a collocated actuator and sensor pair, which provides good stability properties [2]. For example, direct velocity feedback consists of generating a control force proportional to the local velocity of the structure, in which the controller design is reduced to tuning the feedback gain. Engels et al. [3] showed that a decentralised direct velocity feedback control system provides remarkable performance when compared to the equivalent Linear Quadratic Gaussian regulator. This inspired the work presented in this paper on the possibility of having a self-contained control unit, which can be tuned locally, and it can be adopted as modular model for a decentralised control system.

In the literature, other active damping strategies have been considered. For instance, Positive Position Feedback (PPF) controllers efficiently reduce a targeted resonant peak by introducing a second-order filter [4]. The limitation of the PPF filter is that the targeted frequency has to be known accurately, and small changes in the dynamics of the structure can deteriorate the performance of the control system dramatically. Also, control of different modes requires the design of several PPF filters, where the cross coupling between different filters has to be taken into account. Alternatively, Integral Resonant Control (IRC) provides a more robust technique by adding a portion of the actuator signal to the sensor signal, but the suppression of higher order modes is limited to its first order filter behaviour, and the tuning of the feedback gain requires the analysis of the poles of the system, and hence the global dynamics of the plate in our case [5]. Other studies investigated the use of a force feedback [6], acceleration feedback [7, 8] or more complex controllers [9] to improve the stability properties of the system compensating for the phase delay introduced by the actuator.

Piezoelectric actuators [10-12] can be used to implement active damping on lightly damped structures. However, because of their dynamic behaviour, the feedback loop is not unconditionally stable any longer, but a limit in the maximum stable gain is introduced. For example, when piezoelectric patches are used, a delay is present between the velocity measured at the centre of the patch and the bending moment generated at the edge of the patch. The combination of this phase delay and the large amplitudes at high frequencies of the open-loop transfer function makes the control system only conditionally stable [13]. Furthermore, piezoelectric patched do not efficiently excite flexural modes of structures at low frequencies, and relatively large control efforts are required to obtain the desired damping action [12,14]. Alternatively, voice coil actuators [2, 4, 6, 15-25] can better excite flexural modes of structures at low frequencies, generating a point force control excitation with a high force/weight ratio [13]. For this reason, they have been widely used to control vibration in floors [9, 26], aircraft structures [27], instruments for aerospace applications [28], buildings [29], and bridges [30].

Depending on the design, two types of voice coil actuators can be adopted: reactive and inertial. For reactive actuators, the magnet or the coil is mounted on the structure. For inertial actuators, the two components are assembled together in a self-contained unit. The reactive actuator better approximates the ideal skyhook damping, as it generates a constant force with respect to the current input. Consequently, the response of the structure between the control force and the collocated velocity is proportional to the driving-point mobility of the structure, and thus the feedback system is unconditionally stable. On the other hand, such arrangement cannot be always implemented, as it requires a rigid mounting frame to react off. Conversely, the inertial actuator can be directly attached to the structure, as it reacts off the inertial mass. The main drawback of such a solution is that a phase lag in the control force occurs below the first resonance of the inertial actuator, which makes the control system only conditionally stable [2], and hence limits the performance.

The performance of a local velocity feedback control unit largely depends on the feedback gain. However large values of feedback gain do not guarantee the global minimisation of vibration, as shown in Fig. 1. As the feedback gain increases, the total vibration of a plate reduces down to a minimum, but for further increase of the feedback gain, a pinning condition is approached, in which new structural resonances rise at higher frequencies because of the change in the boundary conditions. Alternative methods have been suggested to tune the feedback gain, such as maximising the absorbed power by the control unit from the structure [1,31]. This optimisation has been shown to almost correspond to the vibration minimisation when a finite structure with reactive actuators is considered [1, 3133]. The two methods are analytically equivalent for a single-degree-of-freedom structure using inertial actuator [34]. However, the method has not been addressed for distributed systems coupled with inertial actuators.

Nevertheless, if an inertial actuator is used for the feedback control, the control system may approach instability for gains lower than the optimal one, which minimises the global vibration. The stability limit depends on the choice of 


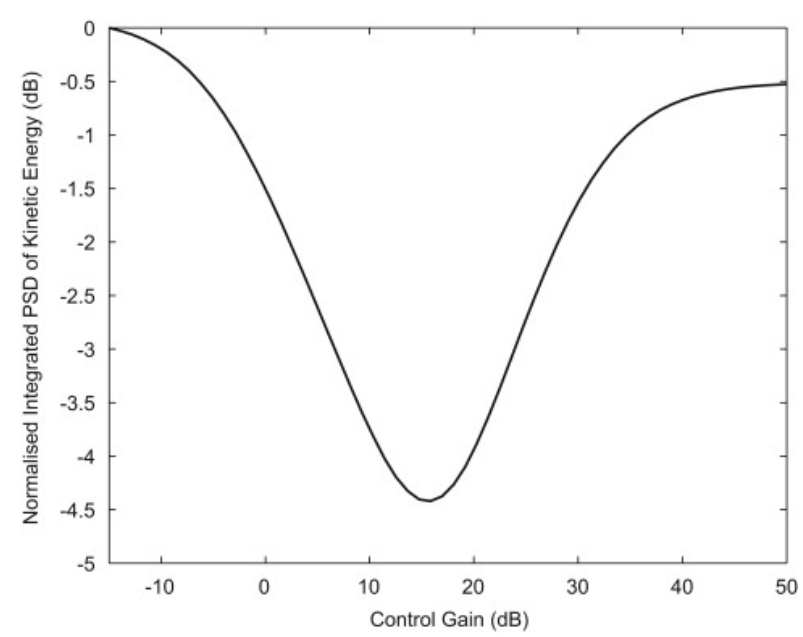

(a)

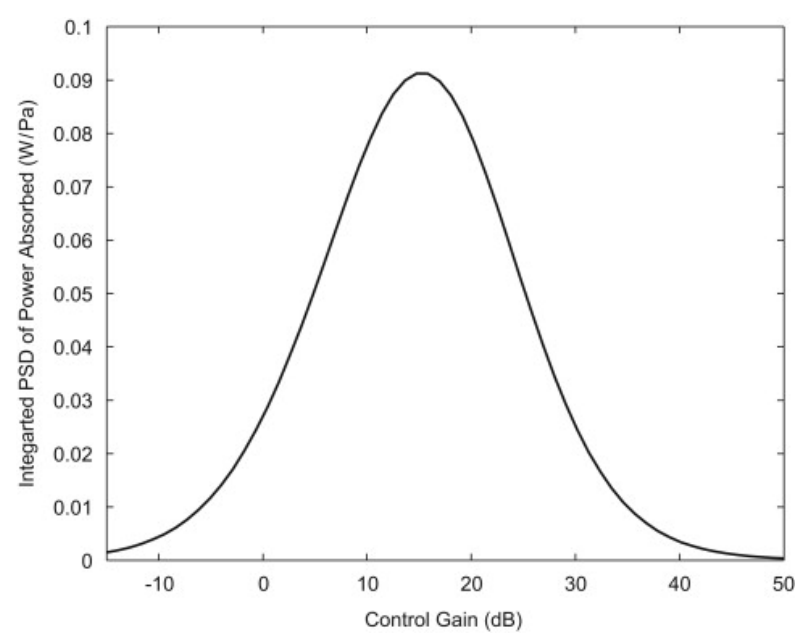

(b)

Figure 1: (a) Ratio of the $1 \mathrm{~Hz}-1 \mathrm{kHz}$ frequency-integrated PSD of the plate total kinetic energy with and without control as function of the control gain. (b) $1 \mathrm{~Hz}-1 \mathrm{kHz}$ frequency-integrated PSD of the absorbed power by the feedback loop from [1].

the plate-actuator pair, which can directly affects the performance of the active control. A first attempt to improve the stability requires the inertial actuator to have the natural frequency well below the natural frequency of the structure, and to have high damping ratio [2]. This led to specific studies on the design of inertial actuators for velocity feedback control of lightweight structures, in which different assembly options were investigated, in such a way to obtain a relatively large suspended mass on soft springs [22]. A common issue of such a design is the large static displacement of the moving mass, which, in presence of shocks, may cause stroke saturation [21]. Different solutions to this problem have been investigated, such as the implementation of an inner feedback loop on the displacement [23, 24], or the control force [6]. Alternatively, a compensator can be designed to counterbalance the phase lag of the inertial actuator [17]; or an inerter flywheel can be added to the actuator, to increase the apparent mass without increasing the static displacement of the moving mass [25].

This paper presents a comprehensive study on the effect of the 'inertial actuator-plate' pair in a local velocity feedback control unit for vibration reduction. The aim is to contrast the performance of the control system with an ideal skyhook damping control in terms of maximum reduction of global vibration in the structure, and to find the tuning of the velocity feedback gain. The analysis is expressed as $H_{2}$-norm optimal control, and is discussed both in frequency domain and time domain. A similar study has already implemented a frequency domain formulation and a time domain formulation [32]. However, an ideal velocity feedback control was considered, and the influence of the frequency range of interest of the active control was not addressed. This paper, instead, describes both the plate and the actuator dynamics, and analyses the effect of the frequency range considered in the optimisation to determine the optimal gain.

The contribution of this paper is organised as follows. First, the dynamics of the control system is introduced, and a novel formulation of the absorbed power by the control unit from the structure is presented. Second, the performance of an inertial actuator is contrasted with an ideal velocity feedback. The study is enriched with parametric studies, which provide a general guidance on the choice or design of an inertial actuator control unit with respect to the plate. The analysis is carried out with respect to non-dimensional parameters, such as: the frequency ratio $v$ between the actuator resonant frequency and the first resonant frequency of the plate; the actuator internal damping $\zeta_{a}$; the mass ratio $\mu$ between the plate and the actuator; and the ratio $r$ of the moving mass of the actuator, with respect to its total mass. Finally, an experimental validation is presented, in which the reduction of kinetic energy and the absorbed power by the inertial actuator are measured on a plate subjected to a point excitation, and the results are compared with the numerical simulations. In particular, the tuning of the feedback gain is discussed, as well as the influence of the frequency range of integration on the optimisation procedure.

The work is organised in 5 Sections. In Section 2, two mathematical models of the control system are presented, in 
the state space and frequency domain, in which the global dynamic of the control system is obtained assembling two independent subsystems, which describe the structure and actuator dynamic behaviour. In Section 3, the performance of the control system with the inertial actuator is investigated in the frequency domain, in terms of the total kinetic energy and absorbed power by the control unit. A parametric study is carried out in Section 4, and, finally, an experimental validation is presented in Section 5, in which a digital feedback control system is implemented on an aluminium plate with nine accelerometers to estimate the kinetic energy.

\section{Mathematical model}

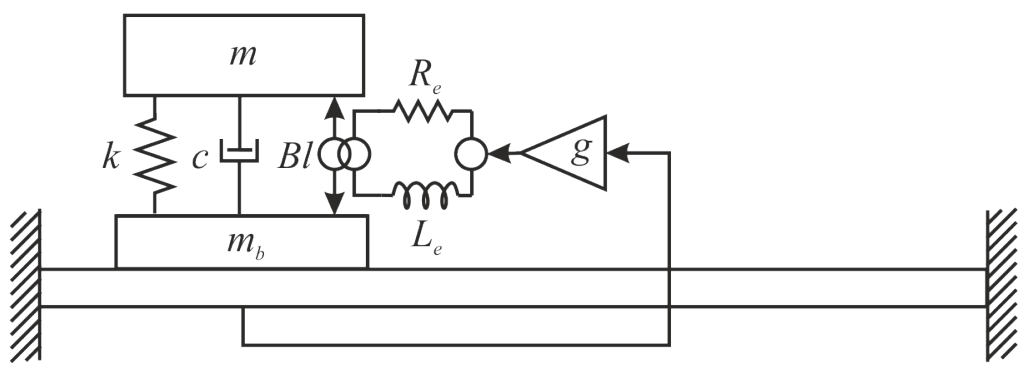

Figure 2: Fully clamped plate with a local velocity feedback control loop by means of an inertial actuator.

A fully clamped rectangular plate excited by a rain-on-the-roof excitation is considered. A local velocity feedback is used, in which an inertial actuator produces a current proportional to the local velocity of the plate, as shown in Fig. 2. The dynamics of the velocity feedback controlled system is derived, in which the plate and actuator dynamics couple together. The stability of the velocity control system is discussed, and finally, two different methods to obtain the time-averaged kinetic energy and the time-averaged absorbed power are derived.

The geometrical and physical properties of the aluminium plate are summarised in Table 1. A random control position on the plate is chosen, to avoid nodal lines of the first structural modes. The parameters of the inertial actuator are obtained through identification of the Micromega ${ }^{\circledR}$ IA01, commercially available, and are summarised in Table 2.

\begin{tabular}{llrl}
\hline Parameter & & Value & \\
\hline Planar dimensions & $l_{x} \times l_{y}$ & $800 \times 500$ & $\mathrm{~mm}^{2}$ \\
Thickness & $h$ & 2 & $\mathrm{~mm}$ \\
Density & $\rho$ & 2720 & $\mathrm{~kg} / \mathrm{m}^{3}$ \\
Young's modulus & & $7 \times 10^{10}$ & $\mathrm{~N} / \mathrm{m}^{2}$ \\
Poisson ratio & & 0.33 & \\
Internal damping ratio & $\zeta$ & 0.01 & \\
Control position & $\left(x_{c}, y_{c}\right)$ & $\left(0.31 l_{x}, 0.41 l_{y}\right)$ & \\
\hline
\end{tabular}

Table 1: Geometry and physical parameters of the plate.

\subsection{Frequency domain formulation}

The kinetic energy of the structure is used to assess the performance of a control system, since it provides information about the global reduction of vibration of the structure. For a thin plate in flexural vibration, subject to stationary and ergodic random excitation, the time-averaged kinetic energy $\bar{K}_{e}$ of a plate, with velocity $\dot{w}$, density $\rho$, and constant thickness $h$ can be written as presented in $[35,36]$

$$
\bar{K}_{e}=E\left[\frac{1}{2} \int_{S} \rho h \dot{w}^{2}(x, y, t) d S d t\right]=\frac{1}{2 \pi} \int_{-\infty}^{\infty} S_{K} d \omega,
$$




\begin{tabular}{lcrl}
\hline Parameter & & Value & \\
\hline Moving mass & $m$ & 36 & $\mathrm{~g}$ \\
Base mass & $m_{b}$ & 53 & $\mathrm{~g}$ \\
Natural frequency & $f_{a}$ & 8.7 & $\mathrm{~Hz}$ \\
Damping ratio & $\zeta_{a}$ & 0.3 & \\
Voice coil coefficient & $B l$ & 1.6 & $\mathrm{~N} / \mathrm{A}$ \\
Electrical resistance & $R_{e}$ & 3 & $\Omega$ \\
Electrical inductance & $L_{e}$ & 100 & $\mu \mathrm{H}$ \\
\hline
\end{tabular}

Table 2: Physical properties of the inertial actuator, taken from the datasheet for the Micromega ${ }^{\circledR}$ IA01 transducer.

where $E$ is the expectation operator, and

$$
S_{K}=E\left[\int_{A} \frac{1}{2} \rho h|\dot{w}(x, y)|^{2} d A\right],
$$

where $A$ is the plate surface, and $S_{K}$ is commonly referred as Power Spectral Density (PSD) of the kinetic energy. More precisely, $S_{K}$ is the spatial integral of the power spectral density of the velocity of the structure, weighted with the local mass of the structure. However, for simplicity, it will be referred to "kinetic energy PSD" throughout the paper.

Because of the orthogonality of the eigenfunctions with respect to the mass-operator, the kinetic energy PSD can be rewritten with respect to the plate modal components $\boldsymbol{\Omega}$ as [1]

$$
S_{K}=\frac{1}{2} m_{p} \operatorname{Tr}\left[\mathbf{\Omega} \mathbf{\Omega}^{H} \mathbf{S}_{\mathbf{f f}}\right]
$$

where $m_{p}$ is the mass of the plate, $H$ is the Hermitian operator, and $\mathbf{S}_{\mathbf{f f}}$ is a fully populated matrix containing the PSDs of the modal excitations:

$$
\mathbf{S}_{\mathbf{f f}}=E\left[\int_{S} \int_{S^{\prime}} \boldsymbol{\phi}(x, y) \boldsymbol{\phi}^{T}\left(x^{\prime}, y^{\prime}\right) f_{p}(x, y) f_{p}\left(x^{\prime}, y^{\prime}\right) d S d S^{\prime}\right] .
$$

Here $\phi(x, y)$ is the $N \times 1$ plate eigenvector, $f_{p}$ is the primary force distribution, and $T$ indicates the transpose. In particular, in the case of a rain-on-the-roof excitation, a spatially uniform and uncorrelated distribution of point forces is considered, and $\mathbf{S}_{\mathbf{f f}}$ reduces to the identity matrix [32].

The absorbed power $\bar{P}$ by the control unit from the structure can be obtained from the actuator dynamics, which can be modelled using an electro-mechanical model [16, 37, 38], as shown in Fig. 3. A control force $f_{c}$ is generated when (i) a base velocity $\dot{w}$, or (ii) an electrical input, either a current $i$ or a voltage $e$, are provided. Thus the actuator dynamics can be conveniently described by the two-port network equations

$$
\begin{aligned}
f_{c} & =T_{i} i-Z_{m o} \dot{w}, \\
e & =Z_{e b} i-T_{i} \dot{w}
\end{aligned}
$$

where $Z_{m o}$ is the open-loop mechanical impedance of the inertial actuator for a base excitation, $Z_{e b}$ is the blocked electrical impedance seen by the electrical system when a current excitation is present, and $T_{i}$ is the transduction function of the force transmitted to the ground generated by a current flowing, or, equivalently, an induced voltage generated by a base movement.

The time-averaged absorbed power $\bar{P}$ by the actuator from the structure can be written as the product between the control force $f_{c}$ and the local velocity $\dot{w}_{c}$ as

$$
\bar{P}=E\left[-f_{c}^{T} \dot{w}_{c}\right]=\frac{1}{2 \pi} \int_{-\infty}^{\infty} S_{P} d \omega
$$

with

$$
S_{P}=-E\left[\mathfrak{R}\left\{f_{c}^{H} \dot{w}_{c}\right\}\right]=E\left[\mathfrak{R}\left\{Z_{m o}+T_{i} g\right\}\left|\dot{w}_{c}\right|^{2}\right],
$$

where $g$ is the feedback gain, and $S_{P}$ is the real part of the cross PSD of the control force and the local velocity of the structure, and, for simplicity, it will be referred to as "absorbed power PSD" throughout the paper. 


\section{Reactive actuator}

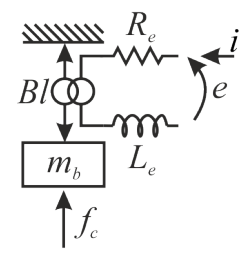

$$
Z_{m o}=j m_{b} \omega
$$$$
T_{i}=B l
$$$$
Z_{c b}=R_{e}+j L_{c} \omega
$$

Inertial actuator

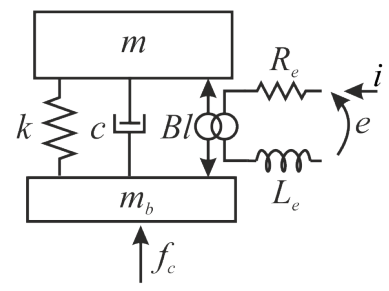

$Z_{m o}=j m_{b} \omega+\frac{j m \omega(k+j c \omega)}{k+j c \omega-m \omega^{2}}$

$T_{i}=\frac{-m B l \omega^{2}}{k+j c \omega-m \omega^{2}}$

$Z_{e b}=R_{e}+j L_{e} \omega+\frac{j B l^{2} \omega}{k+j c \omega-m \omega^{2}}$

Figure 3: Schematic representation of a reactive and inertial actuator, with the respective open-loop mechanical impedance $Z_{m o}$, force per unit current $T_{i}$ and electrical blocked impedance $Z_{e b}$.

\subsection{Time domain formulation}

A time domain formulation can be written, which avoids the integration required in the frequency domain. For time domain formulation, the controlled-system dynamics is introduced in the state-space as

$$
\left\{\begin{array}{l}
\dot{\mathbf{x}}=\mathbf{A}_{g} \mathbf{x}+\mathbf{B}_{\mathbf{d}} \mathbf{u}_{\mathbf{d}} \\
\mathbf{y}=\mathbf{C x}
\end{array}\right.
$$

in which $\mathbf{A}_{g}$ includes the plate dynamics and the effect of the actuator velocity feedback, as derived in the formulation provided in Eq. (A.18) in Appendix A. The state-vector $\mathbf{x}$ contains the modal velocities and displacements of the uncoupled plate, and the velocity and displacement of the actuator moving mass; $\mathbf{u}_{\mathbf{d}}$ corresponds to the external disturbance; and the output $\mathbf{y}$ is the modal velocity vector. The output matrix $\mathbf{C}$ returns the modal velocities of the plate, and it is equal to $\mathbf{C}=\left[\begin{array}{ll}\mathbf{0}_{N \times N} & \mathbf{I}_{N \times N}\end{array}\right]$ when a reactive actuator is used, and to $\mathbf{C}=\left[\begin{array}{llll}\mathbf{0}_{N \times N} & \mathbf{I}_{N \times N} & \mathbf{0}_{N \times 2}\end{array}\right]$, when an inertial actuator is used. The complete derivation of the state-space formulation starting from the independent plate and inertial actuator sub-systems can be found in the Appendix A.

According to Eq. (8), the time-averaged kinetic energy $\bar{K}$ can be rewritten as [32]

$$
\bar{K}_{e}=E\left[\frac{1}{2} \int_{S} \rho h \mathbf{x}^{T}(t) \mathbf{C}^{T} \phi^{2}(x, y) \mathbf{C} \mathbf{x}(t) d S\right]=\frac{1}{2} m_{p} \operatorname{Tr}\left[\mathbf{C}^{T} \mathbf{C X}\right],
$$

where $\mathbf{C}$ is the selection matrix of the modal velocities of the plate, and $\mathbf{X}$ is the covariance matrix of the steady-state response [35], which satisfies the Lyapunov equation [4]:

$$
\mathbf{A}_{g} \mathbf{X}+\mathbf{X} \mathbf{A}_{g}^{T}=-\mathbf{B}_{\mathbf{d}} \mathbf{F} \mathbf{B}_{\mathbf{d}}{ }^{T},
$$

with

$$
\mathbf{F}=E\left[\int_{S} \int_{S^{\prime}} \boldsymbol{\phi}(x, y) \boldsymbol{\phi}^{T}\left(x^{\prime}, y^{\prime}\right) f_{p}(x, y) f_{p}\left(x^{\prime}, y^{\prime}\right) d S d S^{\prime}\right],
$$

which is the covariance matrix of the plate primary excitation, and for a rain-on-the-roof excitation, it reduces to the identity matrix, as discussed in Eq. (4).

Similarly, the time-averaged absorbed power by the control unit from the structure can be written, from Eq. (6) as

$$
\bar{P}=E\left[-f_{c}^{T} \dot{w}_{c}\right]=-\operatorname{Tr}\left[\mathbf{C}_{v}^{T} \mathbf{C}_{f} \mathbf{X}\right],
$$


in which $\mathbf{C}_{v}$ and $\mathbf{C}_{f}$ are, respectively, the output matrices of the state-space model for the velocity of the structure at the control position, and for the control force generated by the actuator, as presented in Appendix A.

\section{Spectral analysis}

The effect of a velocity feedback on the plate with an inertial actuator can be investigated in the frequency domain in terms of the kinetic energy PSD and absorbed power PSD, which are obtained from Eq. (3) and Eq. (7).

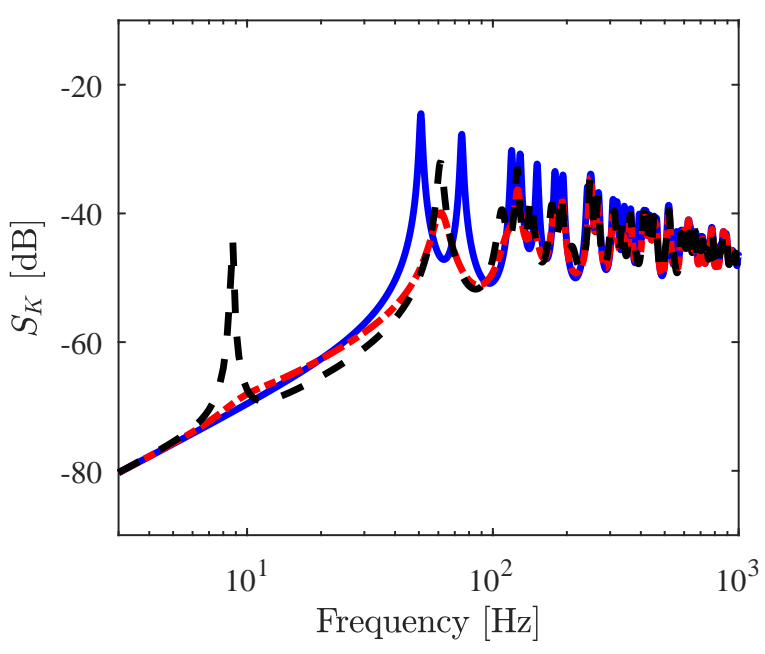

(a)

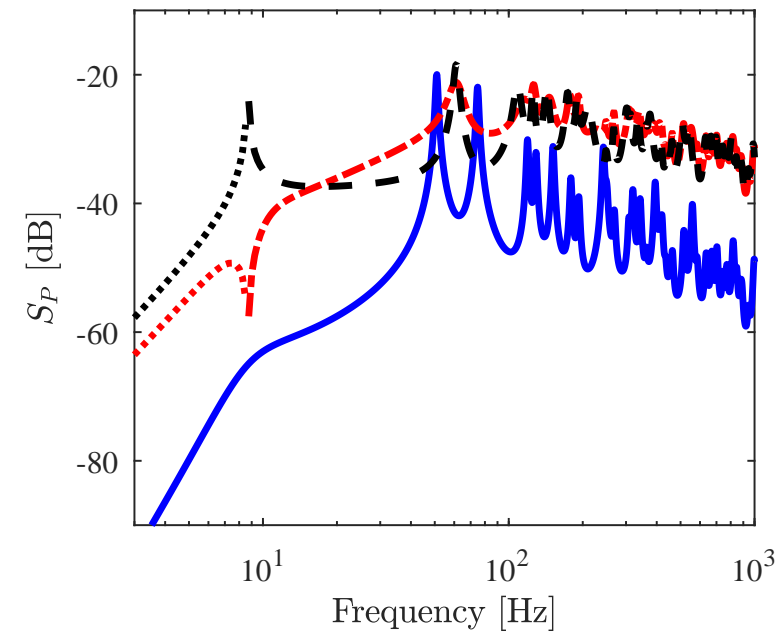

(b)

Figure 4: (a) Kinetic energy PSD and (b) absorbed power PSD of a velocity feedback control system with an inertial actuator for different values of feedback gain $g$. Uncontrolled configuration in solid blue, minimum time-averaged kinetic energy in dash-dotted red, and unstable configuration in dashed black. Negative absorbed power depicted with dotted lines. (For interpretation to the references to colour in this figure, the reader is referred to the web version of this article.)

Fig. 4a shows the kinetic energy PSD. In the open-loop configuration, when the inertial actuator is mounted on the plate but the feedback gain is zero, the plate dynamics is characterised by well separated resonant peaks. However, as the frequency increases, the magnitude of the peaks globally decreases, and the plate dynamic response approaches the infinite plate configuration, in which the plate behaves as a damper [13, 39]. Also, at high frequencies, the modal overlap increases, and so the dynamic response is increasingly determined by the mass-type response of the non-resonant terms rather than by the resonant terms. For this reason, the benefits of active damping is reduced [40].

Active damping increases in the plate by increasing the feedback gain, which reduces the first resonant peaks amplitude. However, such a control system differs from the active damping using reactive actuator for two reasons: (i) spillover at low frequency, and (ii) instability before the pinning condition. The spillover at low frequencies is due to the $180^{\circ}$ phase lag of the inertial actuator force per unit current, which occurs below the resonance of the inertial actuator, at $8.7 \mathrm{~Hz}$. Consequently, as the feedback gain increases, a new peak rises at the actuator resonance as shown in Fig. 4a, and eventually reaches infinite magnitude, when the maximum stable gain of $513 \mathrm{As} / \mathrm{m}$ is approached. The upper limit in the feedback gain prevents the control system from approaching the pinning condition. More importantly, depending on the choice of the plate-actuator pair, the stability limit may be lower than the optimal gain, which minimises the kinetic energy, and in this case, rather poor performance can be achieved.

Fig. $4 \mathrm{~b}$ shows the absorbed power PSD of the actuator from the plate. In the open-loop configuration, the inertial actuator acts passively, and thus the absorbed power is positive throughout all the frequency range, with peaks corresponding to the plate resonances. For large values of feedback gain, the plate resonances reduce in magnitude, and the absorbed power is more evenly distributed over the entire frequency range. However, as the feedback gain increases, the phase of the control force below the actuator resonance is delayed with the phase lag of $180^{\circ}$ due to the actuator force per unit current. Consequently, below the actuator resonance, the absorbed power is negative, which 
means that the power is injected by the actuator into the structure, as confirmed by the spillover in the kinetic energy PSD.

\section{Parametric study}

In this section the influence of different parameters on the performance of a local velocity feedback control unit is discussed. First the effect of active damping for vibration suppression on a plate is presented, with respect to the plate dimensions. Secondly, the coupling between the plate and an inertial actuator is investigated, in such a way to provide some general rules for the design of an actuator to reduce vibration on a specific plate, or, conversely, determine the possible applications of a given actuator.

The objective of this study is to determine:

- the effect of the plate dimensions on the control system in terms of maximum reduction of vibration and optimal gain;

- the influence of the actuator parameters on the performance of the control system, with respect to the plate, and in comparison with an ideal sky-hook damping.

\subsection{Plate dimensions and control position}

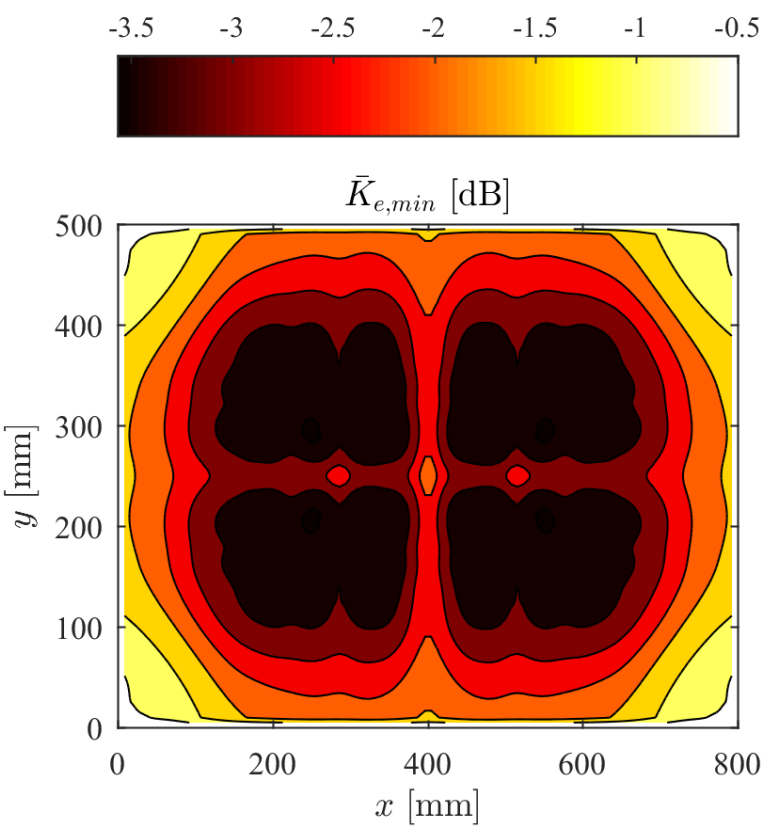

(a)
100
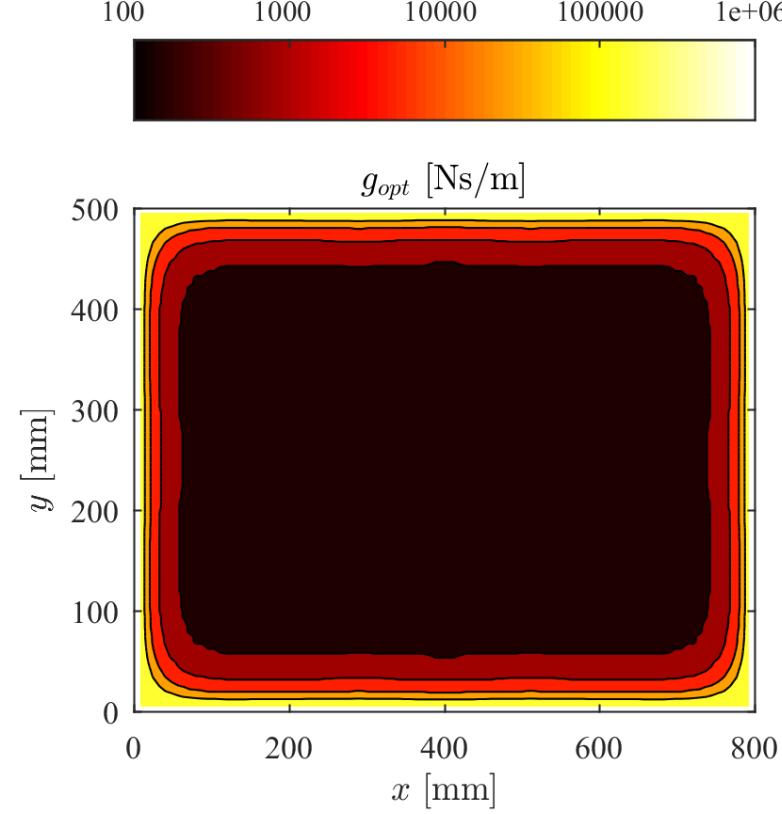

(b)

Figure 5: (a) Normalised minimum time-averaged kinetic energy and (b) relative optimal feedback gain for an ideal skyhook damper for different control positions on the plate. The normalisation is with respect to the kinetic energy of the uncontrolled plate. Frequency range considered is up to $500 \mathrm{~Hz}$.

In order to limit the analysis of the performance of active control on the plate properties, an ideal reactive actuator is considered, in which an ideal control force proportional to the local velocity of the structure is generated. An aluminium plate with constant material properties is considered. Therefore, the density $\rho$, the Poisson ratio, the Young modulus $E$, and the internal damping are assumed to be constant. Consequently, the analysis here focuses on 
the plate dimensions, and on the control position. As a reference, the geometrical and physical properties of the plate are listed in Table 1.

The optimisation of the actuator position plays a crucial part in the design of a control system, as it depends on the control objectives and on the primary disturbance [40]. In this work, the global reduction of kinetic energy is assumed as control objective. Fig. 5a shows the maximum reduction of the kinetic energy at each position on the plate, when an ideal reactive actuator is considered. Because the rain-on-the-roof disturbance excites equally all the structural modes, the performance of the control unit depends purely on the coupling between the structural modes and the control unit. In particular, reductions in the kinetic energy up to $3 \mathrm{~dB}$ are achieved when the nodal lines of the plate are avoided. However, as the control position approaches the edges of the plate, the mobility of the structure reduces, and thus larger values of feedback gain are required. This is confirmed in Fig. 5b, where the optimal gain at different positions is represented.

The influence of the dimensions of the plate on the performance of the active control is analysed using the time domain formulation of Eq. (9), in which only the first 10 modes are considered. This enables to compare different plates, which have resonances at different frequency ranges. Otherwise, if a maximum frequency of integration were defined, a different number of uncontrolled modes would have been included in the integration for different plate sizes, and the effect of the plate dimensions could not be correctly investigated, as discussed in Appendix B.

The results are shown in Fig. 6, with constant control position with respect to the plate dimensions. The planar dimension of the plate does not affect the reduction of kinetic energy neither in amplitude, nor in the optimal gain, as depicted in Fig. 6a. This is because a change in the surface of the plate simply scales the system, and thus the optimal and the pinning conditions do not vary. In this study, the length of the plate changes and the ratio between the length and the width of the plate is kept constant, so that the control position does not change with respect to the nodal lines, and the analysis of the performance of active control depends only on the planar dimension of the plate. Conversely, increasing the thickness of the plate $h$, the optimal gain moves to larger values, as shown in Fig. $6 \mathrm{~b}$. This is due to the driving-point mobility of the plate, which is reduced as the thickness $h$ increases, and therefore a larger control force is required to reduce the plate vibration.

\subsection{Inertial actuator coupling}

In this section the performance of an inertial actuator for vibration reduction is investigated. In particular, a comparison is carried out with the ideal reactive actuator in terms of maximum reduction of kinetic energy, and optimal gain.

Inertial actuators for active control on smart structures are typically required to be light. Nevertheless, Elliott $e t$ al. [2] showed that a low natural frequency of the inertial actuator improves the stability of the feedback control system. This implies low values of internal stiffness in the actuator, and consequently an increase in the stroke of the moving mass. Finally, the control force is proportional to the moving mass of the actuator, and thus the mass ratio between actuator and plate can affect the performance.

For this purpose, it is convenient to rewrite the mechanical impedance and the force per unit current of the inertial actuator as a function of dimensionless parameters, such that

$$
T_{i}=\frac{B l s^{2}}{s^{2}+2 \zeta_{a} \omega_{a} s+\omega_{a}^{2}},
$$

and

$$
Z_{m o}=m_{a} \frac{(1-r) s^{3}+2 \zeta_{a} \omega_{a} s^{2}+\omega_{a}^{2} s}{s^{2}+2 \zeta_{a} \omega_{a} s+\omega_{a}^{2}},
$$

in which $m_{a}=m+m_{b}$ is the total mass of the inertial actuator including the base mass $m_{b}$ and moving mass $m, r$ is the ratio of the moving mass and the total mass of the actuator, $\omega_{a}$ and $\zeta_{a}$ are the actuator natural frequency and damping ratio, respectively. Finally, two other parameters are introduced: the mass ratio $\mu$ between the total mass of the actuator and that of the plate, and the frequency ratio $v$ between the actuator resonant frequency and the first natural frequency of the plate.

The maximum stable gain can be determined through the Nyquist Criterion, and is related to the resonance of the inertial actuator, at which a shift in phase of $180^{\circ}$ occurs [2]. Therefore, the stability limit is expected to depend 


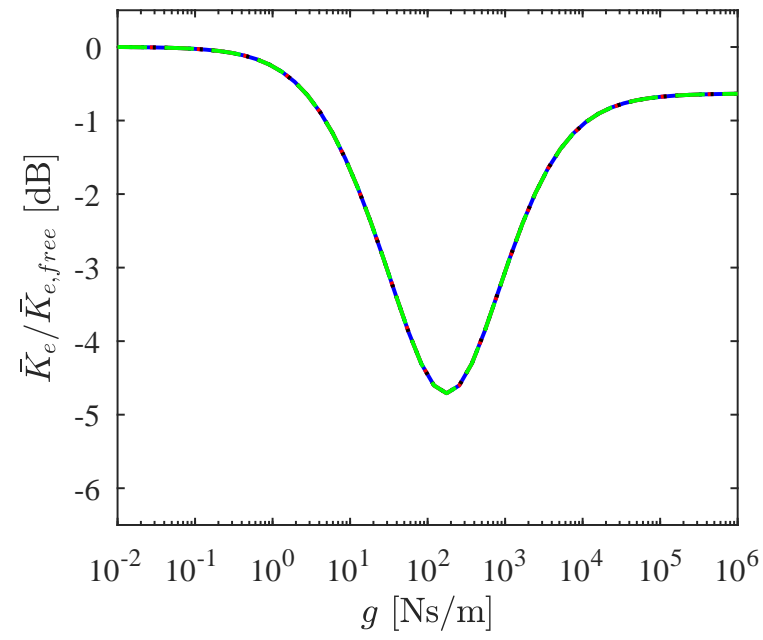

(a)

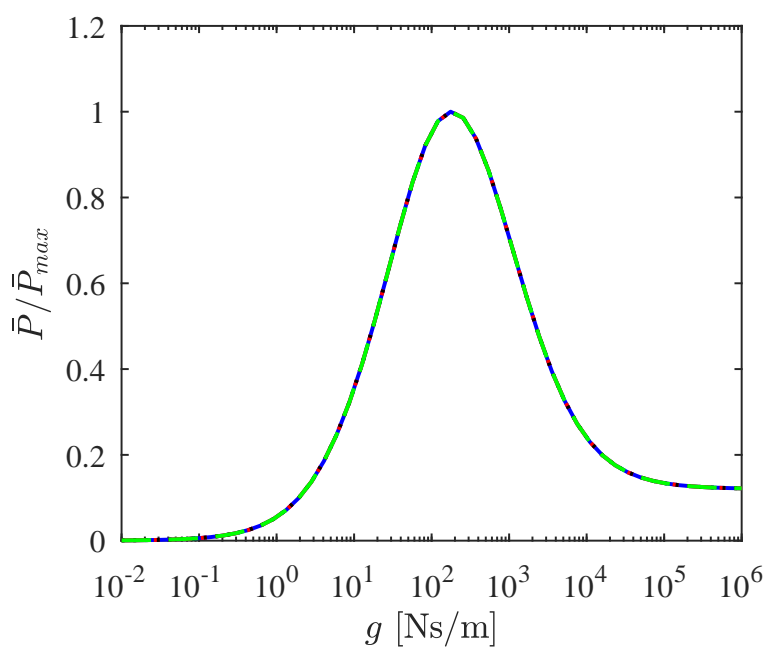

(c)

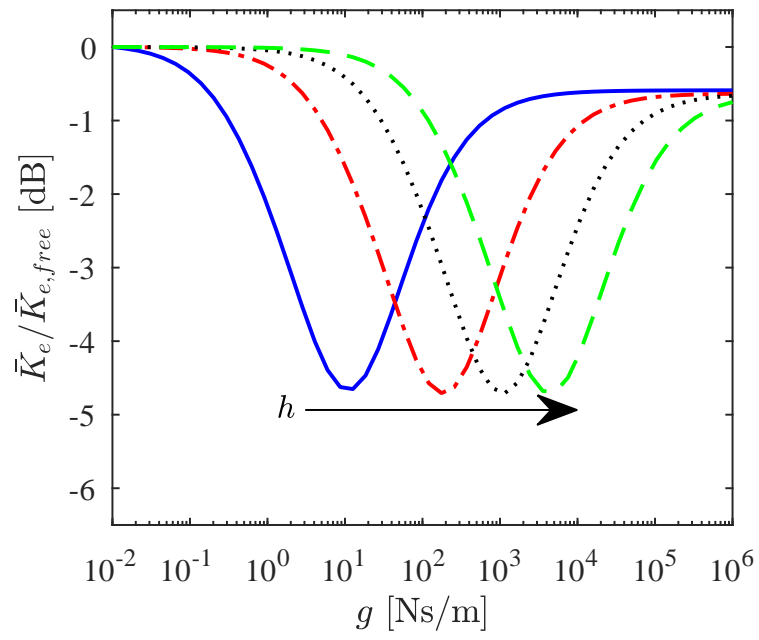

(b)

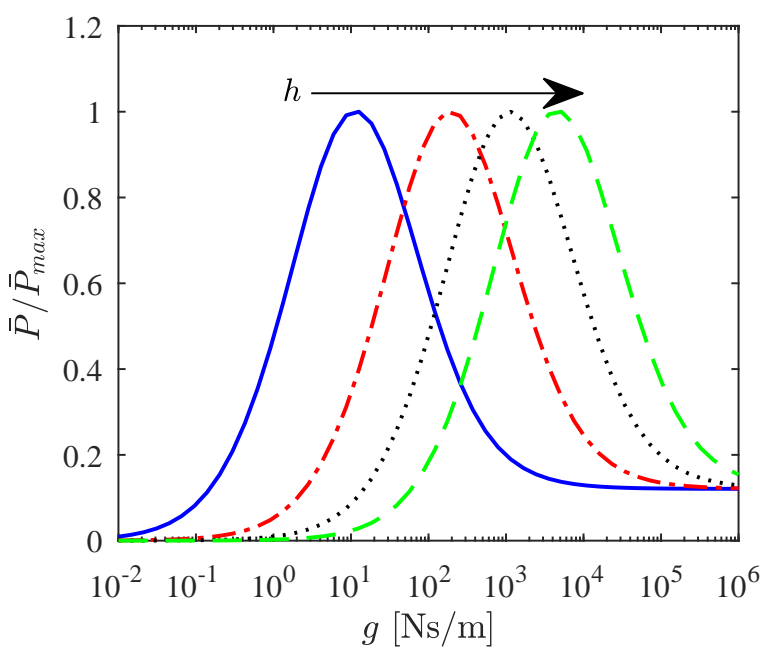

(d)

Figure 6: Time-averaged (a,b) kinetic energy of the plate controlled by using skyhook damping and (c,d) absorbed power by the control unit, when the first 10 modes are considered. For $(\mathrm{a}, \mathrm{c})$ different values of plate size, and for $(\mathrm{b}, \mathrm{d})$ different values of plate thickness $h$, have been used with the same relative position of the control force. Plate lengths $l_{x}$ in (a,c) are: $450 \mathrm{~mm}$ (solid blue), $600 \mathrm{~mm}$ (dash-dotted red), $800 \mathrm{~mm}$ (dotted black), 1 $\mathrm{m}$ (dashed green). Plate thicknesses $h$ in (b,d) are: $0.5 \mathrm{~mm}$ (solid blue), $2 \mathrm{~mm}$ (dash-dotted red), $5 \mathrm{~mm}$ (dotted black), $10 \mathrm{~mm}$ (dashed green). The arrow direction shows the increase of the parameter. (For interpretation to the references to colour in this figure, the reader is referred to the web version of this article.)

mainly on the internal damping $\zeta_{a}$ and the frequency ratio $v$, whereas the other two parameters $\mu$ and $r$ mostly affect the plate dynamics at the first resonance, and thus the performance of the control unit.

In order to obtain an adequate variation for each of these parameters, the results in Fig. 7 are obtained assuming the physical and geometrical properties of the plate and actuator listed, respectively, in Tables 1 and 2 . Therefore, the starting values of the parameters are $\zeta_{a}=0.3, v=0.17, \mu=0.04$ and $r=0.38$. Each parameter is then changed, while keeping the others fixed, and this represents a change in the physical properties of the actuator.

This is further investigated in Fig. 8, in which the maximum stable gain is depicted with respect to the four dimensionless parameters. The gain margin highly depends on the frequency ratio $v$ and the internal damping of the actuator $\zeta_{a}$, as shown in Fig. 8a. However, the stability of the control system is very insensitive to changes in the 


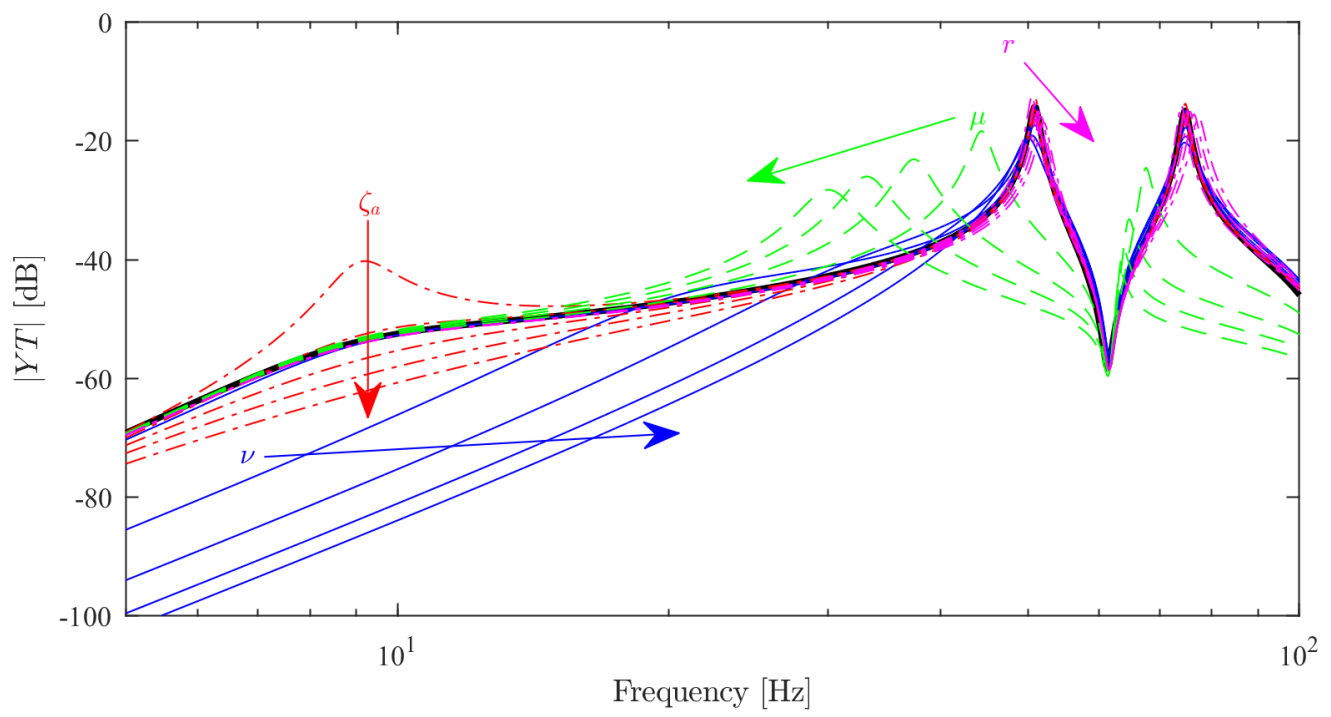

Figure 7: Open-loop transfer function of the velocity feedback control system with an inertial actuator for different values of frequency ratio $v$ (solid blue), actuator damping ratio $\zeta_{a}$ (dash-dotted red), mass ratio $\mu$ (dashed green), actuator mass ratio $r$ (dash-dotted magenta). The arrow direction shows the increase of the parameter. (For interpretation to the references to colour in this figure, the reader is referred to the web version of this article.)
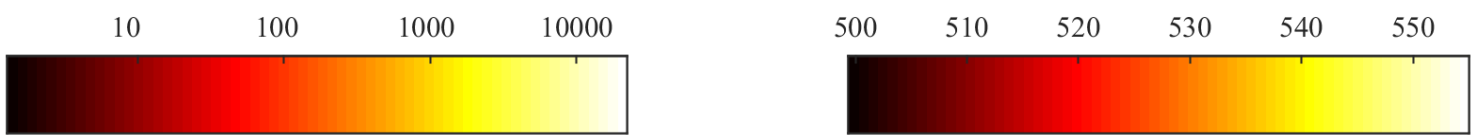

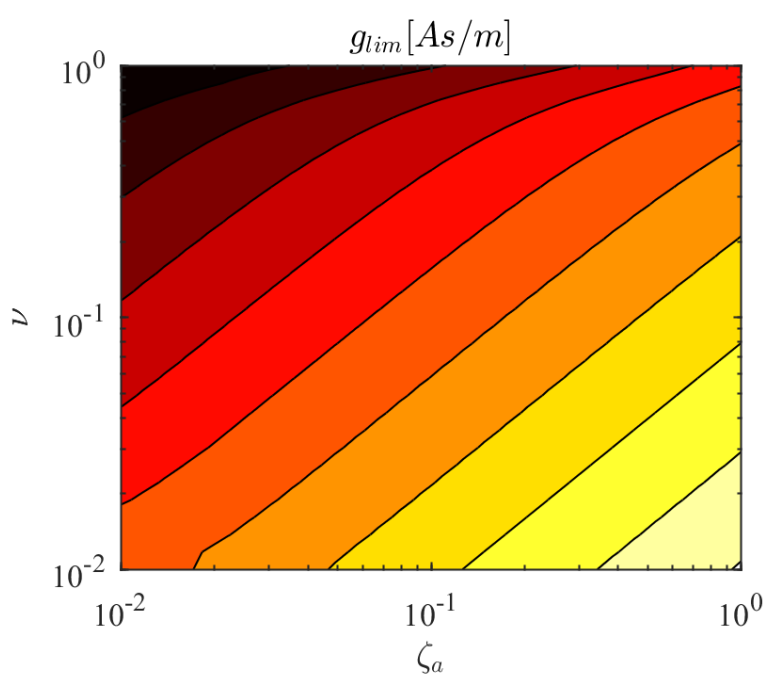

(a)

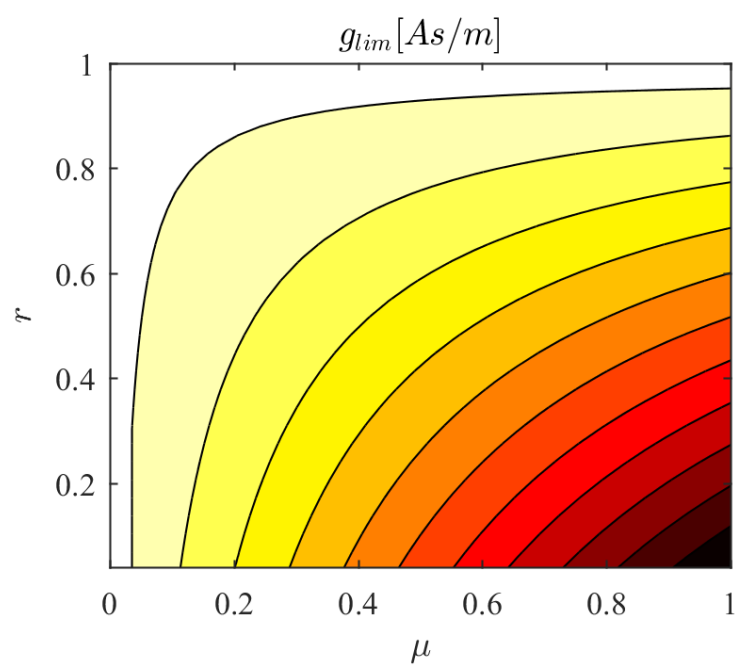

(b)

Figure 8: Maximum stable gain with respect to (a) the actuator damping coefficient $\zeta_{a}$ and the frequency ratio $v$; and (b) the mass ratio $\mu$ and the percentage of the actuator moving mass $r$. Note that the range of feedback gains in (b) is much smaller than in (a). 
mass ratios, as these mostly affect the first resonance of the plate, as depicted in Fig. 8b. Nevertheless, for active control, a low $\mu$ is required, so that the actuator is light with respect to the structure to be controlled. Secondly, a high $r$ is usually recommended, as the control force depends on the moving mass of the inertial actuator. Finally, it is important to notice that in these results, the same plate analysed in Fig. 5 has been considered, for which the optimal gain is about $100 \mathrm{Ns} / \mathrm{m}$, assuming control positions not too close to the bounds. If the transduction coefficient $B l$ of the inertial actuator is considered, the optimal gain, with reference to the driving current, is equivalent to $160 \mathrm{As} / \mathrm{m}$. Therefore, combinations of parameters with a maximum stable gain lower than $160 \mathrm{As} / \mathrm{m}$ in Fig. 8 corresponds to control system in which the minimum in the reduction of kinetic energy of the plate cannot be achieved.

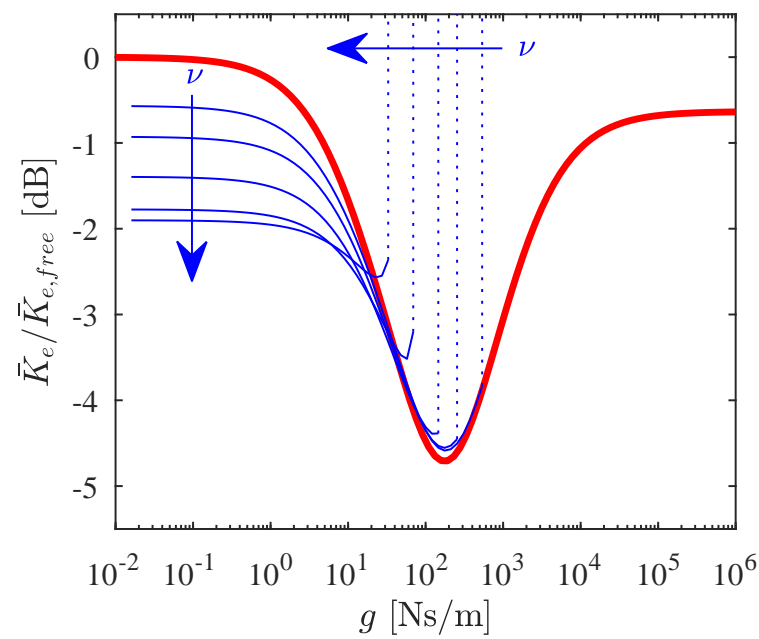

(a)

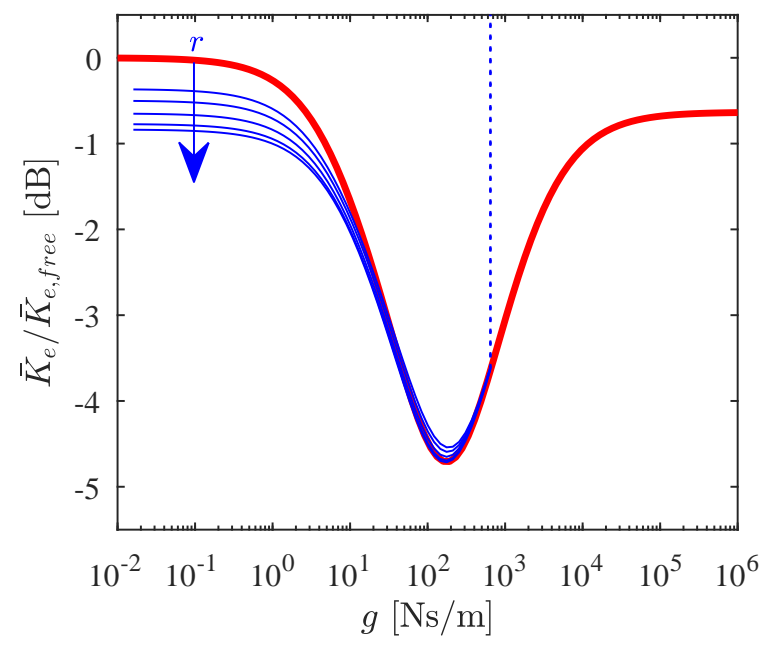

(c)

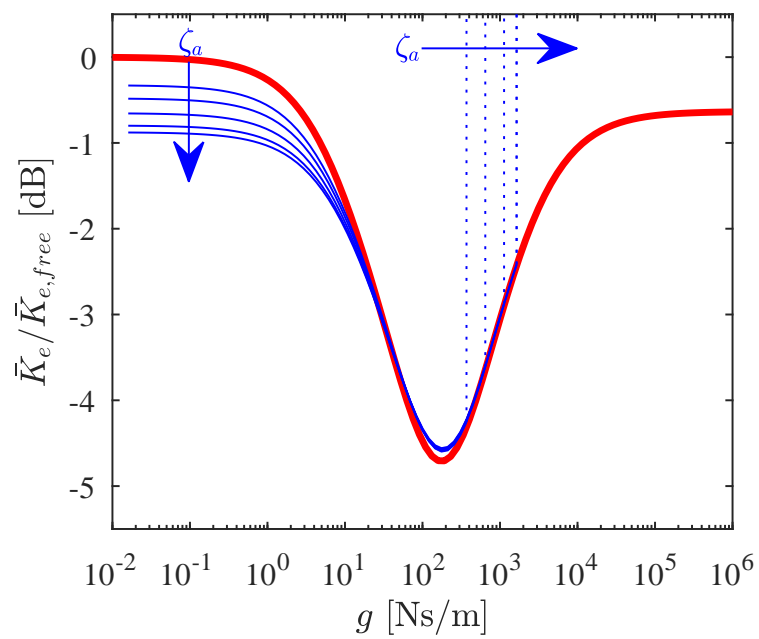

(b)

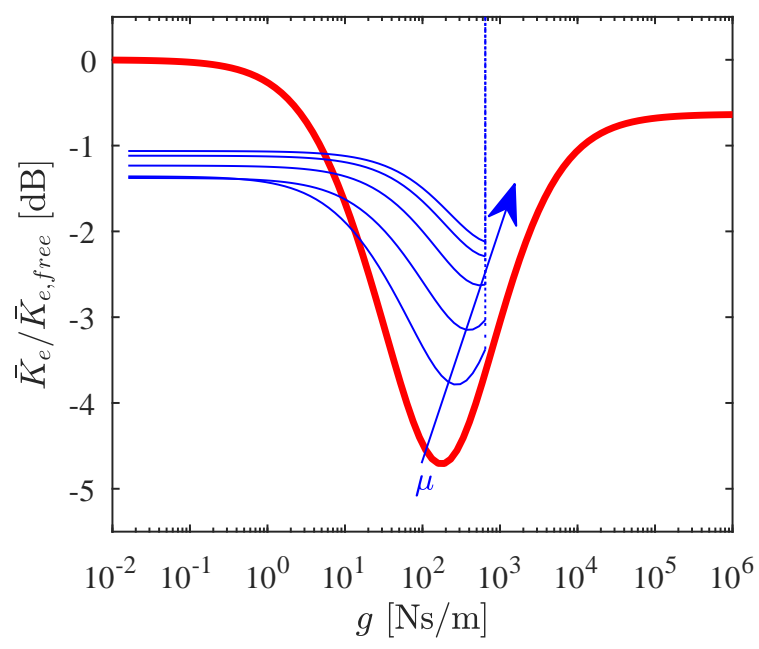

(d)

Figure 9: Kinetic energy of the plate with respect to the velocity feedback gain $g$, changing (a) the frequency ratio $v$; (b) the actuator damping coefficient $\zeta_{a}$; (c) the actuator mass ratio $r$; and (d) the plate-actuator mass ratio $\mu$. Equivalent ideal control force is in thick red, and the fainted blue lines are for increasing values from 0.2 to 1 . The arrow direction shows the increase of the parameter. (For interpretation to the references to colour in this figure, the reader is referred to the web version of this article.)

The influence of the inertial actuator on the reduction of kinetic energy in comparison with an ideal skyhook damping is shown in Fig. 9 and Fig. 10, in which the effect of different inertial actuator parameters on the kinetic energy and the absorbed power, respectively, obtained from the time domain formulation from Eq. (9) and Eq. (12), 


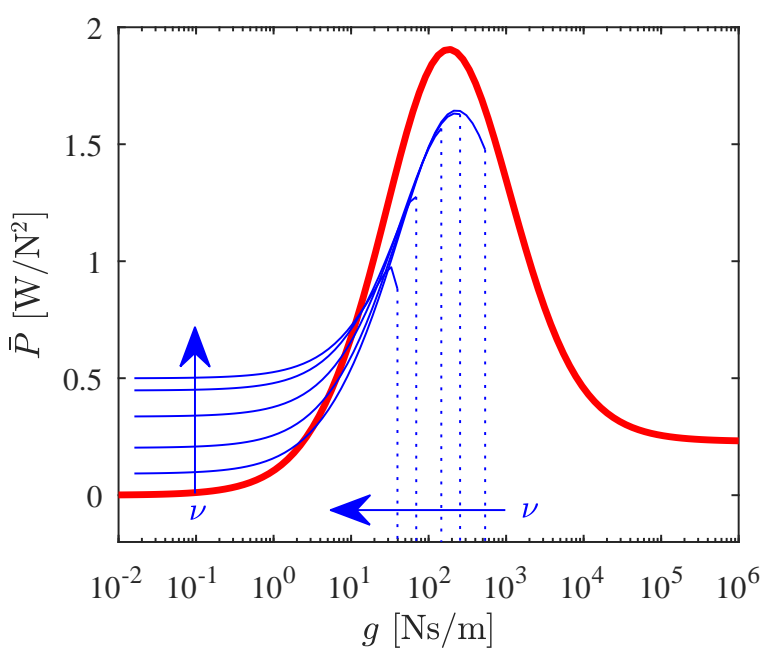

(a)

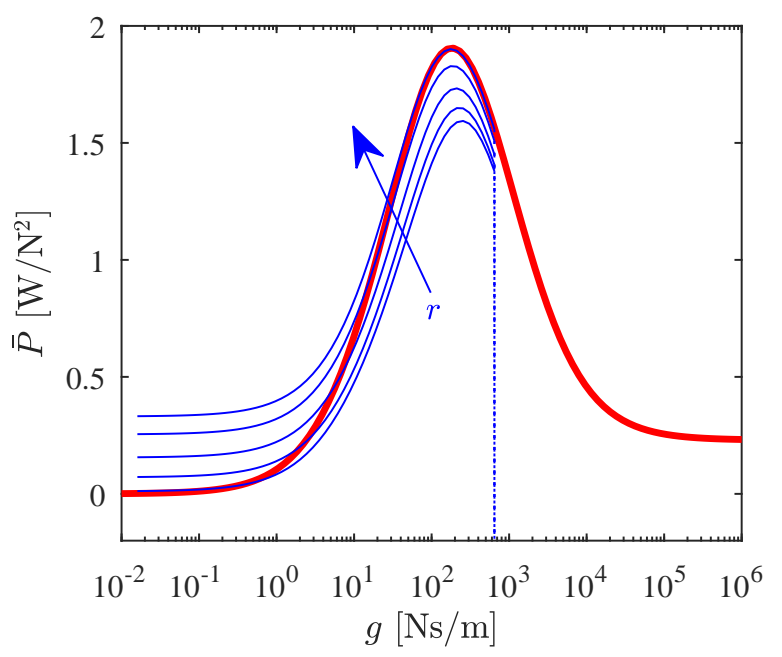

(c)

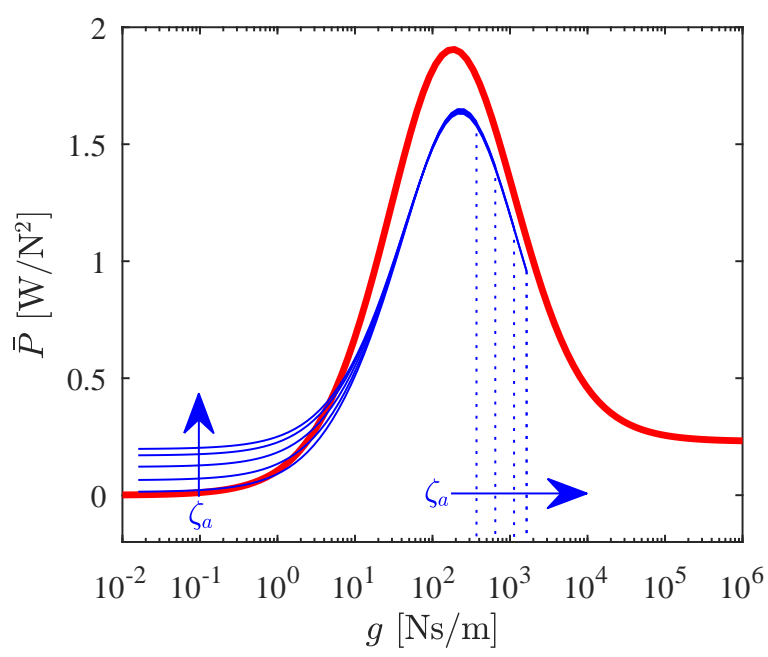

(b)

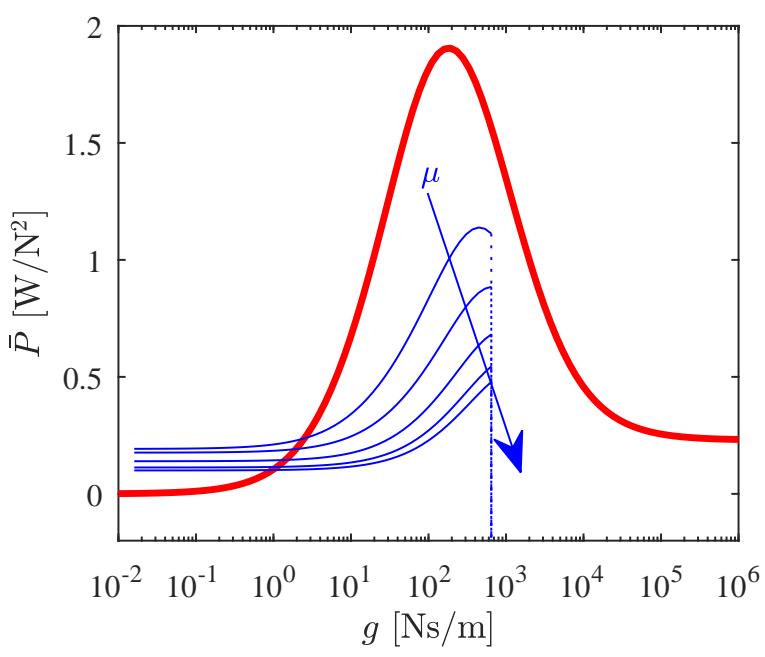

(d)

Figure 10: Absorbed power by the inertial actuator from the plate with respect to the velocity feedback gain $g$, changing (a) the frequency ratio $v$; (b) the actuator damping coefficient $\zeta_{a}$; (c) the actuator mass ratio $r$; and (d) the plate-actuator mass ratio $\mu$. Equivalent ideal control force is in thick red, and the fainted blue lines are for increasing values from 0.2 to 1 . The arrow direction shows the increase of the parameter. (For interpretation to the references to colour in this figure, the reader is referred to the web version of this article.)

are depicted, when the first 10 structural modes are considered. Increasing the frequency ratio $v$, the stability of the control system reduces, and eventually the maximum stable gain reduces to smaller values than the optimal gain, which minimises the kinetic energy. Furthermore, as the frequency ratio grows, the inertial actuator increasingly couples with the first resonance of the plate, and thus more energy is absorbed passively, as depicted in Fig. 10a. Similarly, in Fig. 9b, increasing the damping coefficient $\zeta_{a}$, the maximum stable gain increases, and more reduction is achieved in the kinetic energy for small values of feedback gain.

Conversely, the maximum stable gain does not change significantly with respect to the mass ratios $\mu$ and $r$. As $r$ increases, the actuator moving mass grows accordingly, and the passive effect of the control system is enhanced, as shown in Fig. 9c and 10c. Conversely, if the plate-actuator mass ratio $\mu$ increases, the minimum in the kinetic energy shifts to higher gains, and the control system becomes less effective, as shown in Fig. 9d. 


\section{Experimental validation}

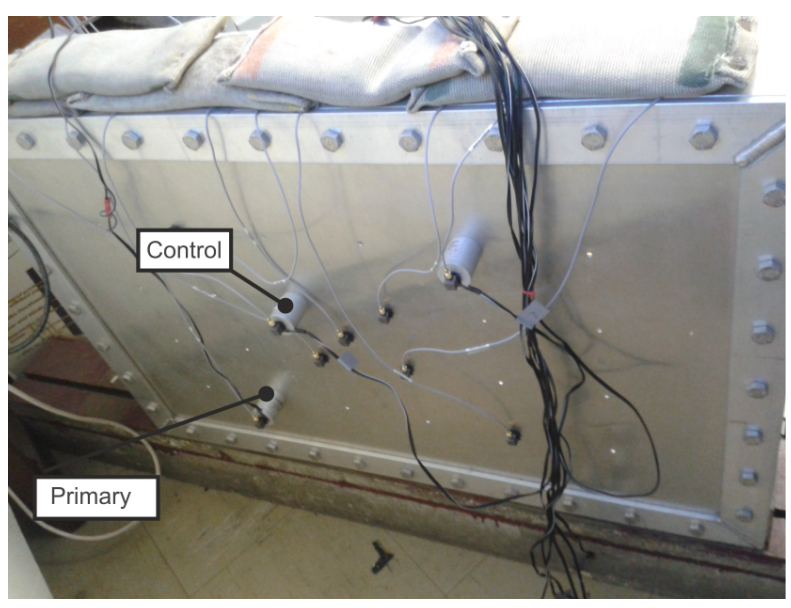

(a)

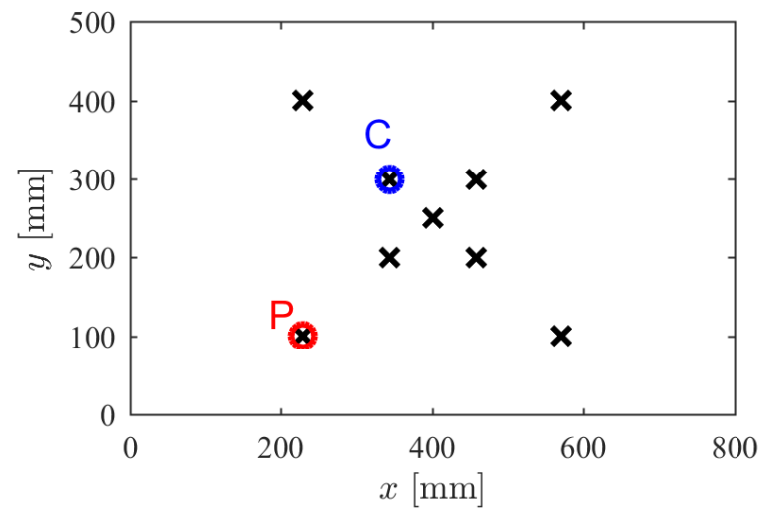

(b)

Figure 11: (a) Experimental setup, and (b) schematic representation of the control system with a primary disturbance (P), a control unit (C), and nine accelerometers indicated with black crosses.

In this section, the tuning of an inertial actuator for vibration control of a plate is investigated experimentally.

Fig. 11 shows the experimental setup, in which a fully clamped aluminium plate is excited by a broadband primary disturbance, and a control unit is used for the velocity feedback control. The kinetic energy is approximated with the sum of the velocity measured at nine sensor positions on the plate, so that all the significant modes of the structure are correctly estimated. Furthermore, the primary disturbance is located in a random position, to avoid nodal lines associated with low frequencies modes.

From Eq. (2), the kinetic energy PSD can be rewritten as

$$
S_{K}=E\left[\int_{S} \frac{1}{2} \rho h|\dot{w}(x, y)|^{2} d S\right] \approx \frac{1}{2} m_{p} \sum_{i=1}^{9}\left|\dot{w}_{i}\right|^{2} .
$$

The absorbed power by the control unit can be measured, from Eq. (6), using the control force and the velocity at the control position. The control force can be obtained, according to Eq. (5a), from the base velocity and the current, once the dynamic behaviour of the inertial actuator is known. The inertial actuator can be fully characterised through two experiments [16], in which the three impedances introduced in Eq. (5a) are measured. In the first experiment, the actuator is rigidly mounted on a shaker and the open-loop mechanical impedance and force per unit current are measured with respect to the imposed base excitation. In the second experiment, the actuator is mounted on a rigid ground and the electrical blocked impedance and force per unit current are measured with respect to an external current flowing through the coil. The identified model can be used to design equivalent digital filters, which provide an estimation of the control force, and hence a real-time estimation of the absorbed power by the control unit.

Fig. 12 shows a comparison between the model and the measurements of the inertial actuator impedances. From the experiments, it can be noticed that only a significant resonance occurs in the frequency range of interest for the study. Therefore, a lumped parameter model is suitable to describe accurately the dynamic behaviour of the actuator. The force per unit current $T_{i}$ is constant in phase and value above the first resonance. However, below the resonance, a phase-shift of $180^{\circ}$ occurs, which reduces the stability margin of the control system. The open-loop mechanical impedance $Z_{m o}$ mostly depends on the two masses of the actuator, the moving magnet and the base mass; the electrical blocked impedance $Z_{e b}$ corresponds to the impedance of a $R L$ circuit, but a peak appears at the actuator resonance, due to the electromagnetic coupling with the mechanical part of the actuator.

Fig. 13 shows a schematic representation of the control scheme [19, 20]. The acceleration of the plate at the nine sensor positions is measured with nine B\&K 4507-B-004 accelerometers together with five B\&K 1704-A-002 CCLD 


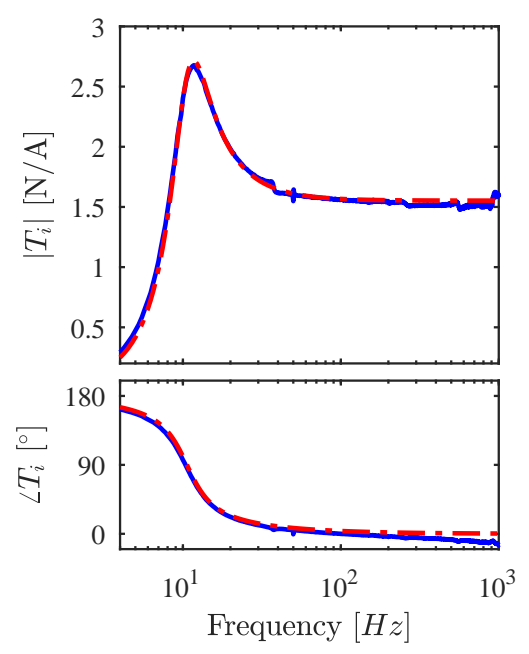

(a)

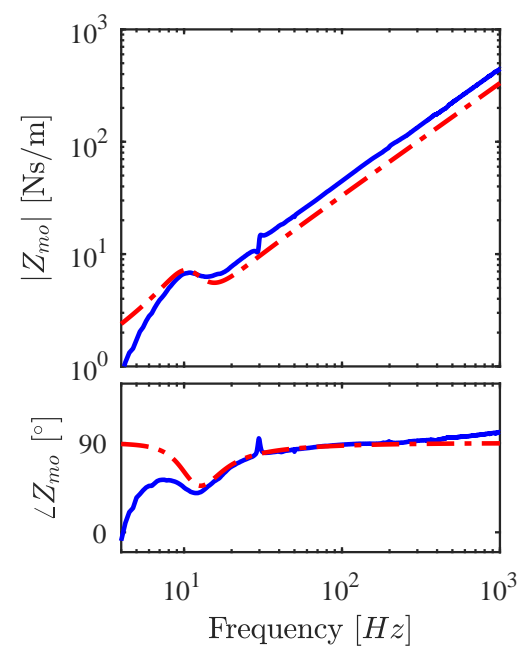

(b)

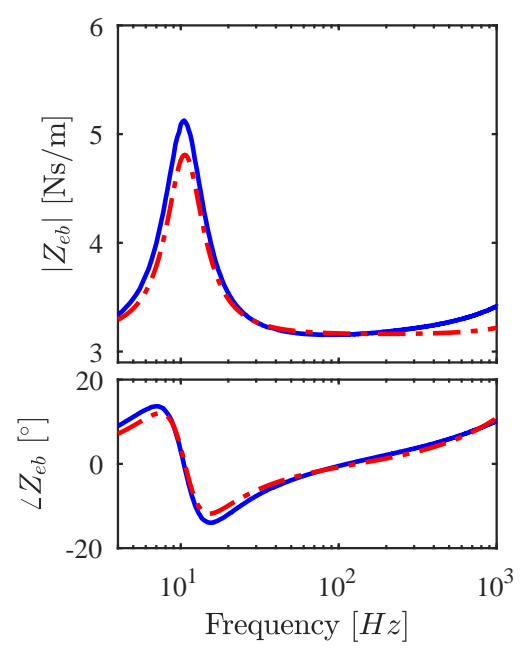

(c)

Figure 12: Measured (solid blue) and theoretical (dash-dotted red) impedances of the Micromega IA01 inertial actuator: (a) force per unit current; (b) mechanical open-loop impedance; and (c) electrical blocked impedance. (For interpretation to the references to colour in this figure, the reader is referred to the web version of this article.)

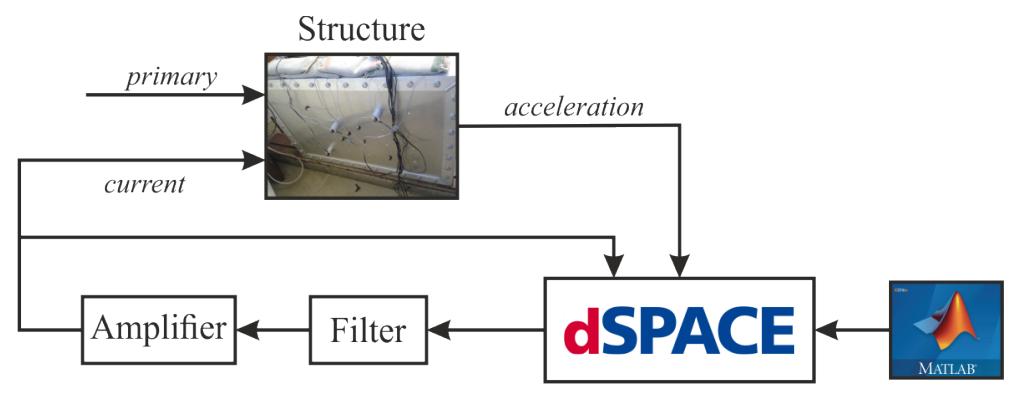

Figure 13: Schematic representation of the control scheme.

signal conditioners, and sent to the $\mathrm{dSPACE}^{\circledR}$ DS1 103 PPC Control Board, running with a sampling frequency, $f_{s}$, of $16 \mathrm{kHz}$, which is configured through the dSPACE ControlDesk ${ }^{\circledR}$ and Matlab Simulink ${ }^{\circledR}$ software. The velocity of the plate at the sensor positions is obtained through digital integration of the measured accelerations. To avoid aliasing at high frequencies, and noise at low frequencies, the signals are filtered with a second order Butterworth digital band-pass filter, with cut-off frequencies of $2 \mathrm{~Hz}$ and $8 \mathrm{kHz}$, before the integration. Finally, a high-pass filter is implemented after the numerical integration to avoid numerical amplification at low frequency.

The feedback control loop consists of a current signal proportional to the local velocity of the structure at the control position. An anti-aliasing low-pass elliptic filter KEMO VBF/23 is then introduced, with a cut-off frequency of $16 \mathrm{kHz}$. The filtered control signal is amplified by a 4-channel current amplifier Micromega, and the output control current is used to drive the Micromega IA01 inertial actuator. Finally, the control current signal is measured to estimate the absorbed power by the inertial actuator for the control.

The primary disturbance is generated by an inertial actuator, driven from the same dSPACE ${ }^{\circledR}$ Control Board, with a white noise, which is filtered with a low-pass elliptic filter KEMO VBF/8, with cut-off frequency of $1 \mathrm{kHz}$. Finally, a third inertial actuator is present on the structure from previous studies, and its passive behaviour has been included in the mathematical model.

The maximum stable gain of the system is obtained through the Nyquist Criterion [41], measuring the open-loop transfer function of the control system, between the digital input control signal generated by the dSPACE ${ }^{\circledR}$ Control 
Board and the output velocity signal at the actuator position. The maximum stable gain is found to be equal to 220 $\mathrm{As} / \mathrm{m}$.

\subsection{Spectral analysis}

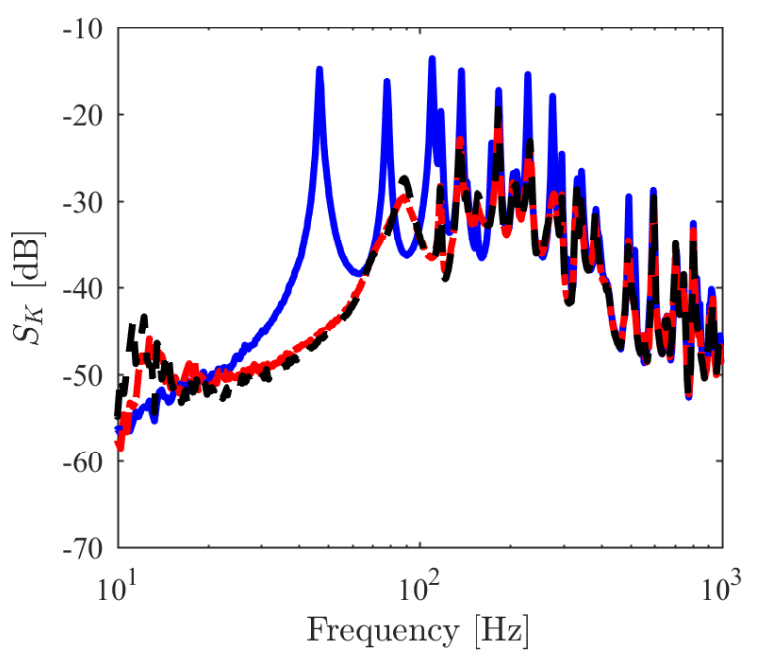

(a)

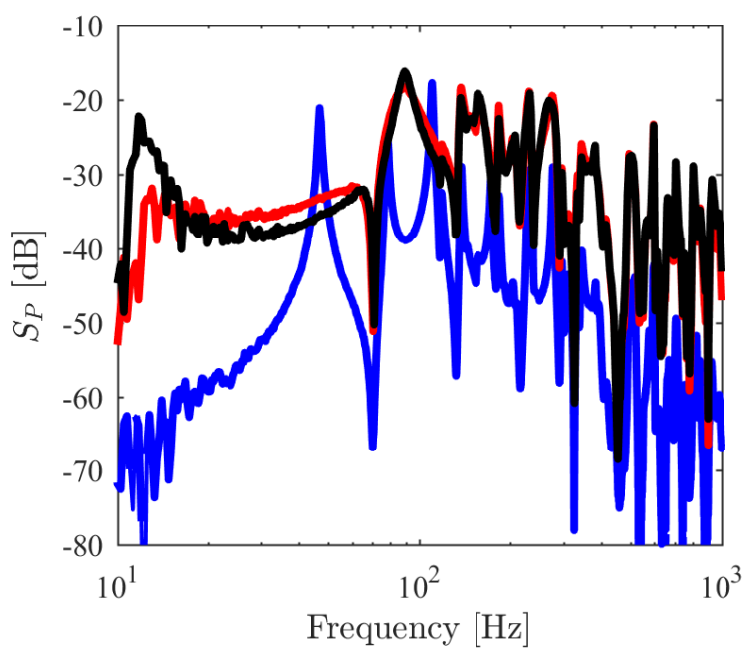

(c)

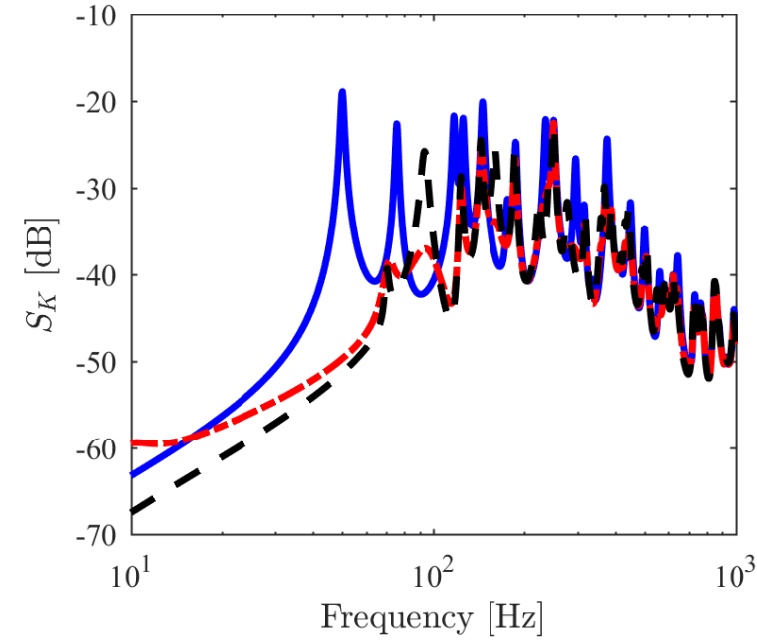

(b)

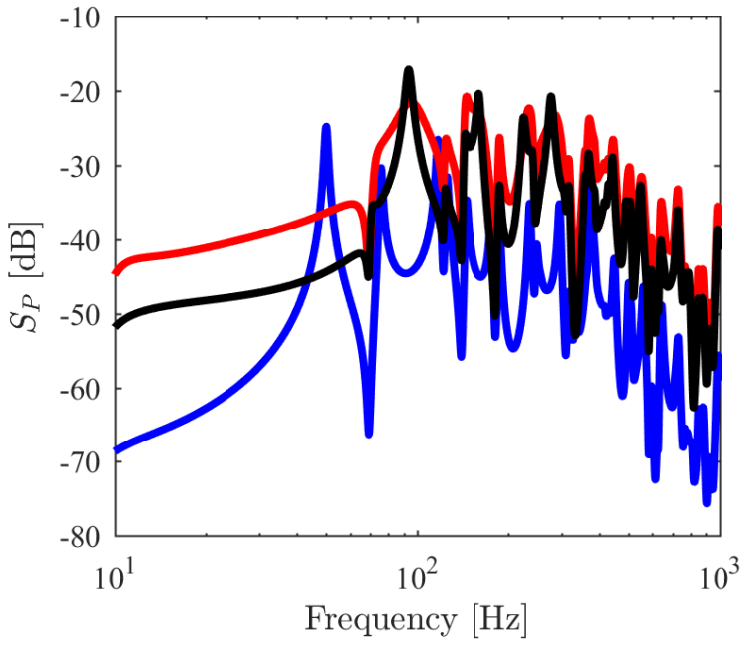

(d)

Figure 14: $(\mathrm{a}, \mathrm{b})$ Kinetic energy PSD and $(\mathrm{c}, \mathrm{d})$ absorbed power PSD of a velocity feedback control system with an inertial actuator for different values of feedback gain $g$. Uncontrolled configuration in solid blue, minimum time-averaged kinetic energy in dash-dotted red, and unstable configuration in dashed black. Experimental results depicted in $(\mathrm{a}, \mathrm{c})$, simulations in $(\mathrm{b}, \mathrm{d})$ (For interpretation to the references to colour in this figure, the reader is referred to the web version of this article.)

The performance of the feedback control is investigated, in the frequency domain, in terms of kinetic energy and absorbed power by the control unit, consistently with Eq. (15) and Eq. (6), respectively. Fig. 14 shows a comparison between measurements and the equivalent mobility/impedance model, for the plate with the passive inertial actuator, for the plate with an optimally tuned active control, and for the plate with active control implementing a feedback gain close to the stability limit. The number and position of the nine accelerometers provide a good estimation of the dynamic response of the plate, in the frequency region of interest up to $1 \mathrm{kHz}$. The inertial actuator is particularly effective in introducing damping in the first structural modes up to $300 \mathrm{~Hz}$, and at higher frequencies smaller effects 
are obtained. Similarly, from a comparison between the measurements and the simulations, it can be noticed that the absorbed power by the control unit is correctly predicted by the numerical model.

In general, the experimental results are in good agreement with the simulations. However, some differences can also be noticed. Although the fully clamped formulation provides a good estimation of the natural frequencies of the plate, the measured resonances occur at slightly lower frequencies because of the non-ideal boundary conditions. At low frequencies, the results are limited to the frequency region above $10 \mathrm{~Hz}$ because of the low frequency noise present in the setup. Furthermore, high frequency delay is introduced in the system by the digital system, which has not been modelled.

The effect of the active damping on the first structural modes is summarised in Table 3, where the reductions of the kinetic energy PSD at the structural resonances below $300 \mathrm{~Hz}$ are listed. The experimental results show that active damping is effective not only at the first structural mode, but also at higher frequencies. Although the dynamics of the plate used in the experiment has not been identified, and it has been assumed to be fully clamped, the numerical model provides a good approximation of the plate dynamics for comparison purposes.

\begin{tabular}{lrrrrrrrrrrrr}
\hline Mode & 1 & 2 & 3 & 4 & 5 & 6 & 7 & 8 & 9 & 10 & 11 & 12 \\
\hline Numerical results: & & & & & & & & & & & & \\
Frequency [Hz] & 50 & 76 & 117 & 125 & 145 & 176 & 187 & 235 & 250 & 296 & 316 & 376 \\
Reduction [dB] & 31 & 17 & 21 & 11 & 7 & 5 & 2 & 8 & 0 & 11 & 2 & 9 \\
& & & & & & & & & & & & \\
Experimental results: & & & & & & & & & & & & \\
Frequency [Hz] & 47 & 78 & 110 & 117 & 137 & 174 & 183 & 210 & 229 & 277 & 297 & 309 \\
Reduction [dB] & 30 & 17 & 23 & 10 & 9 & 8 & 3 & 2 & 10 & 12 & 5 & 4 \\
\hline
\end{tabular}

Table 3: Numerical and measured reduction of kinetic energy associated with the structural modes up to $300 \mathrm{~Hz}$.

\subsection{Time-average}

To better assess the effect of the feedback gain on the control system, the time averaged kinetic energy and absorbed power are analysed for different frequency regions, up to $250 \mathrm{~Hz}, 500 \mathrm{~Hz}$, and $1 \mathrm{kHz}$. The results are shown in Fig. 15. As discussed in Section 4, a larger reduction of kinetic energy is measured, when only the low frequency region up to $250 \mathrm{~Hz}$ is considered. However, the optimal gain does not change with respect to the frequency range of integration. Finally, instability occurs in the measurement at gains lower than the theoretical stability limit, due to the phase delay at high and low frequencies, which have not been included in the model. 


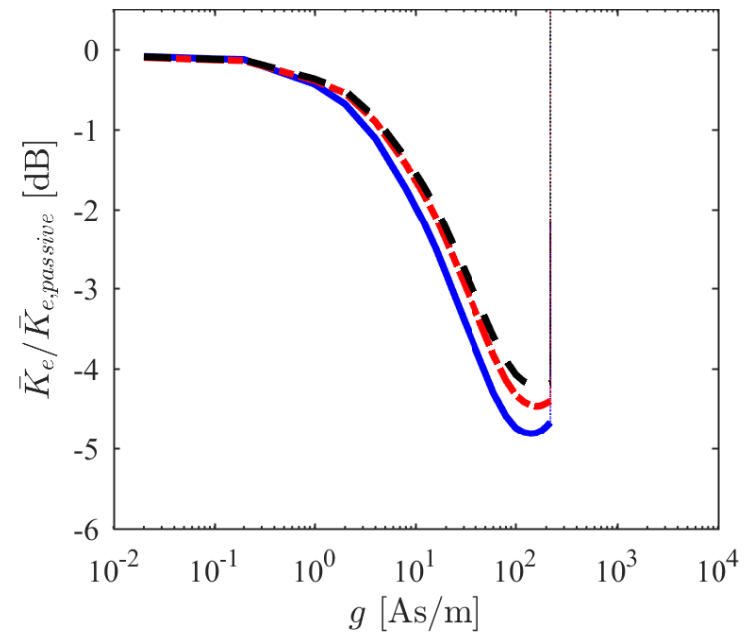

(a)

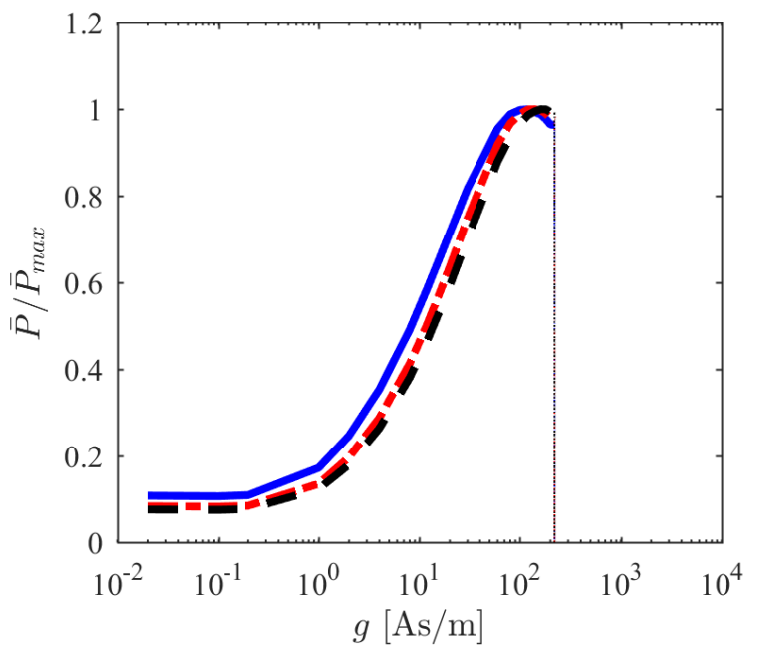

(c)

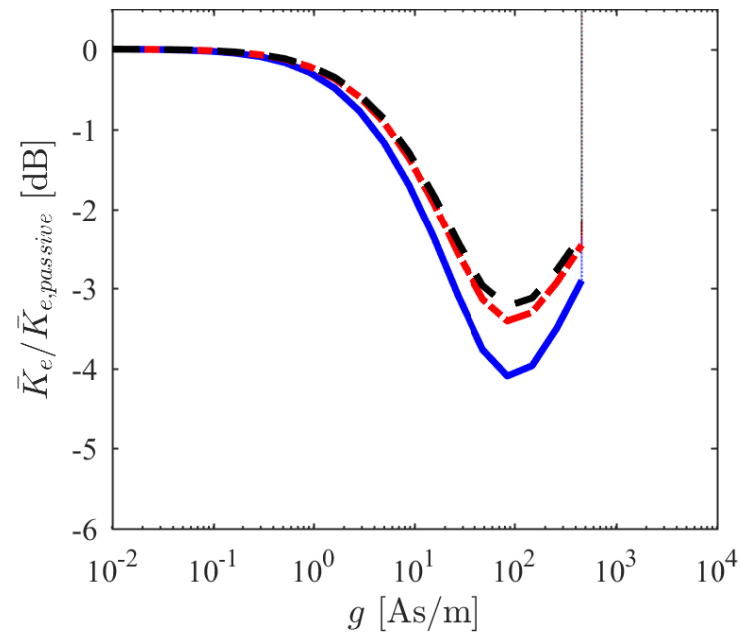

(b)

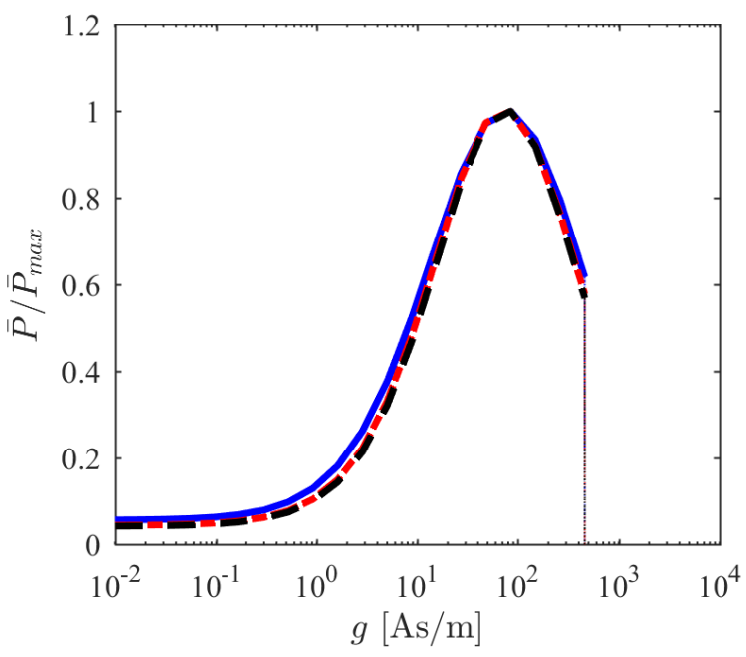

(d)

Figure 15: (a,b) Time-averaged kinetic energy and (c,d) absorbed power with respect to the feedback gain (a,c) measured and (b,d) simulated, for different frequency ranges: up to $250 \mathrm{~Hz}$ (solid blue line), $500 \mathrm{~Hz}$ (dash-dotted red line), and $1 \mathrm{kHz}$ (dotted black line). (For interpretation to the references to colour in this figure, the reader is referred to the web version of this article.)

\section{Conclusions}

This paper presents a theoretical and experimental study on the performance of an inertial actuator for velocity feedback control of a plate. In particular, the effect of different physical parameters of the actuator and of the plate on the vibration reduction are investigated through a parametric study.

The optimal gain, which minimises the kinetic energy, is shown to be independent of the planar dimensions of the plate. However, as the thickness increases, the optimal gain becomes larger, since the plate becomes stiffer.

When an inertial actuator is used, the performance of the control system depends on the dynamic coupling between the actuator and the structure. The reduction of vibration largely depends on the actuator mass. As the inertial actuator becomes heavier, compared to the plate, more energy is absorbed passively, and the benefit of active control reduces. In practice, inertial actuators with large masses behave as poorly tuned mass dampers. Conversely, light actuators approximate an ideal velocity feedback control system well. 
The optimal gain for a local velocity feedback control with an inertial actuator almost corresponds to the one obtained for an ideal control force. This simplifies the control design procedure down to two parameters: the optimal gain and the maximum stable gain. The optimal gain depends on the control position and the plate dimensions. Conversely, the maximum stable gain depends on the actuator choice.

The influence of the frequency range of integration in assessing the reduction of kinetic energy is analysed both numerically and experimentally. The absorbed power by the inertial actuator from the structure is directly estimated from the control current and plate velocity at the control position, through an identified model of the actuator. Reductions up to $5 \mathrm{~dB}$ are measured, when the dynamic response of the system up to $250 \mathrm{~Hz}$ is considered. Increasing the frequency range of integration to $1 \mathrm{kHz}$, the measured reduction of kinetic energy reduces to $4 \mathrm{~dB}$. It is shown that the implementation of a simple local velocity feedback law with an inertial actuator effectively reduces broadband vibration of a plate, and the velocity feedback gain can be tuned locally, through the estimation of the absorbed power.

\section{Acknowledgements}

The authors gratefully acknowledge the European Commission for its support of the Marie Skłodowska-Curie program through the ITN ANTARES Project (GA 606817).

\section{Appendix A. State-space formulation}

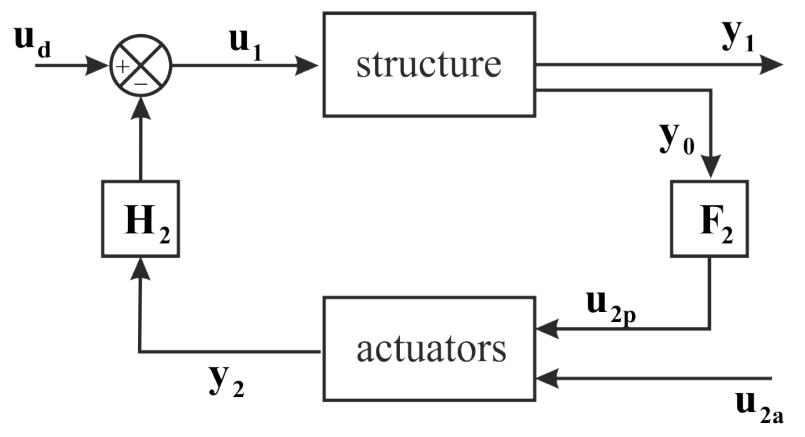

Figure A.16: State-space representation of the coupling between structure and actuators.

In this section the dynamic coupling between a structure and an actuator is discussed in the state-space.

In order to be consistent with the mobility-impedance method presented in Section 2, two independent models for the plate and the actuator are considered as subsystems, and their coupling through a velocity feedback control in a global state-space system is described, according to Ref. [42].

As summarised in Table A.4, the subscript (1) indicates the plate state-space system and the subscript (2) the actuator.

\section{Appendix A.1. Structure and control unit dynamics}

The state-space model of the plate can be derived from Hamilton's variational principle [41], as described in Refs. $[32,36]$ and expressed as

$$
\left\{\begin{array}{l}
\dot{\mathbf{x}}_{1}=\mathbf{A}_{1} \mathbf{x}_{1}+\mathbf{B}_{1} \mathbf{u}_{1} \\
\mathbf{y}_{1}=\mathbf{C}_{1} \mathbf{x}_{1}+\mathbf{D}_{1} \mathbf{u}_{1}
\end{array},\right.
$$




\begin{tabular}{|c|c|c|}
\hline Physical system & Notation & Description \\
\hline Plate & $\begin{array}{l}\mathbf{A}_{1}, \mathbf{B}_{1}, \mathbf{C}_{1}, \mathbf{D}_{1} \\
\mathbf{y}_{1} \\
\mathbf{u}_{1}\end{array}$ & $\begin{array}{l}\text { output: plate modal velocities } \\
\text { input of } \mathbf{B}_{\mathbf{1}} \text { : primary disturbance }\end{array}$ \\
\hline Actuator & $\begin{array}{l}\mathbf{A}_{2}, \mathbf{B}_{2 \mathrm{p}}, \mathbf{B}_{2 \mathbf{a}}, \mathbf{C}_{2}, \mathbf{D}_{2 \mathbf{a}}, \mathbf{D}_{2 \mathbf{p}} \\
\mathbf{y}_{2} \\
\mathbf{u}_{2 \mathbf{p}} \\
\mathbf{u}_{2 \mathbf{a}}\end{array}$ & $\begin{array}{l}\text { output: actuator control force } \\
\text { input of } \mathbf{B}_{\mathbf{2}} \text { and } \mathbf{D}_{\mathbf{2}} \text { : kinematics at the actuator base } \\
\text { input of } \mathbf{B}_{\mathbf{2}} \text { and } \mathbf{D}_{\mathbf{2}} \text { : current control signal }\end{array}$ \\
\hline Plate + Actuator & $\begin{array}{l}\mathbf{A}, \mathbf{B}_{\mathbf{d}}, \mathbf{B}_{\mathbf{c}}, \mathbf{C}, \mathbf{D}_{\mathbf{d}}, \mathbf{D}_{\mathbf{c}} \\
\mathbf{y} \\
\mathbf{u}_{\mathbf{d}} \\
\mathbf{u}_{\mathbf{c}}\end{array}$ & $\begin{array}{l}\text { output: plate modal velocities } \\
\text { input of } \mathbf{B}_{\mathbf{d}} \text { and } \mathbf{D}_{\mathbf{d}} \text { : primary disturbance } \\
\text { input of } \mathbf{B}_{\mathbf{c}} \text { and } \mathbf{D}_{\mathbf{c}} \text { : current control signal }\end{array}$ \\
\hline
\end{tabular}

Table A.4: Description of the state-space systems considered.

in which the $N$ modal displacements an velocities are the state variables $\mathbf{x}_{\mathbf{1}}$, the input $\mathbf{u}_{\mathbf{1}}$ is the modal force, and the output $\mathbf{y}_{\mathbf{1}}$ contains the modal velocities. The state-space matrices are defined as

$$
\begin{aligned}
\mathbf{A}_{\mathbf{1}} & =\left[\begin{array}{cc}
\mathbf{I} & \mathbf{0} \\
-\mathbf{M}_{1}^{-1} \mathbf{K}_{1} & -\mathbf{M}_{1}^{-1} \mathbf{D}_{1}
\end{array}\right], \\
\mathbf{B}_{\mathbf{1}} & =\left[\begin{array}{c}
\mathbf{0} \\
\mathbf{M}_{1}^{-1}
\end{array}\right], \\
\mathbf{C}_{\mathbf{1}} & =\left[\begin{array}{ll}
\mathbf{0} & \mathbf{I}
\end{array}\right], \\
\mathbf{D}_{\mathbf{1}} & =\mathbf{0},
\end{aligned}
$$

in which $\mathbf{M}_{1}, \mathbf{K}_{1}$, and $\mathbf{D}_{1}$ are, respectively, the modal mass, modal stiffness and modal damping matrices, i.e. $N \times N$ diagonal matrices, with the $\mathbf{N}$ modal parameters. Finally, $\mathbf{I}$ and $\mathbf{0}$ are, respectively, identity and zero matrices.

The electro-mechanical dynamics of an inertial actuator can be represented in the state-space as

$$
\left\{\begin{array}{l}
\dot{\mathbf{x}}_{\mathbf{2}}=\mathbf{A}_{\mathbf{2}} \mathbf{x}_{\mathbf{2}}+\mathbf{B}_{2 \mathrm{p}} \mathbf{u}_{\mathbf{p}}+\mathbf{B}_{2 \mathbf{a}} \mathbf{u}_{\mathbf{a}} \\
\mathbf{y}_{\mathbf{2}}=\mathbf{C}_{\mathbf{2}} \mathbf{x}_{\mathbf{2}}+\mathbf{D}_{\mathbf{2}} \mathbf{u}_{\mathbf{p}}+\mathbf{D}_{\mathbf{2}} \mathbf{u}_{\mathbf{a}}
\end{array},\right.
$$

in which, in order to be consistent with the two-port network introduced in Section 2, the output $\mathbf{y}_{2}$ is the control force generated by the transducer; $\mathbf{u}_{\mathbf{p}}$ is the kinematic input from the base mass of the actuator, i.e. the base mass displacement, velocity and acceleration; and $\mathbf{u}_{\mathbf{a}}$ is the electrical input for the active control.

In the current-driven configuration, the actuator state-space model can be rewritten as

$$
\left\{\begin{array}{l}
\dot{\mathbf{x}}=\left[\begin{array}{cc}
0 & 1 \\
-\frac{k}{m} & -\frac{c}{m}
\end{array}\right] \mathbf{x}+\left[\begin{array}{ccc}
0 & 0 & 0 \\
\frac{k}{m} & \frac{c}{m} & 0
\end{array}\right] \mathbf{u}_{\mathbf{p}}+\left[-\frac{B l}{m}\right] \mathbf{u}_{\mathbf{a}}, \\
\mathbf{y}=\left[\begin{array}{ll}
k & c
\end{array}\right] \mathbf{x}+\left[\begin{array}{lll}
-k & -c & -m_{b}
\end{array}\right] \mathbf{u}_{\mathbf{p}}+[B l] \mathbf{u}_{\mathbf{a}}
\end{array}\right.
$$

in which the state variables $\mathbf{x}_{\mathbf{2}}$ are the displacement and velocity of the moving mass, and the other parameters refer to Table 2 .

\section{Appendix A.2. Feedback control system}

In a feedback control system, the structure and actuator state-spaces can be rearranged in a single model as

$$
\left\{\begin{array}{l}
\dot{\mathbf{x}}=\mathbf{A x}+\mathbf{B}_{\mathbf{d}} \mathbf{u}_{\mathbf{d}}+\mathbf{B}_{\mathbf{c}} \mathbf{u}_{\mathbf{c}} \\
\mathbf{y}=\mathbf{C x}+\mathbf{D}_{\mathbf{d}} \mathbf{u}_{\mathbf{d}}+\mathbf{D}_{\mathbf{c}} \mathbf{u}_{\mathbf{c}}
\end{array},\right.
$$


in which the state vector $\mathbf{x}$ includes the two state vectors $\mathbf{x}_{\mathbf{1}}$ and $\mathbf{x}_{\mathbf{2}}$, from the state-space models of the structure and the inertial actuator respectively from Eq. (A.1) and (A.3). The output $\mathbf{y}$ of the system is assumed to be equal to the modal velocities of the structure $\mathbf{y}_{\mathbf{1}}$; and the input of the system are the external disturbance $\mathbf{u}_{\mathbf{d}}$, and the control signals $\mathbf{u}_{\mathbf{c}}$ for the inertial actuator. With reference to Fig. A.16, the coupling equations between the two state-space models can be summarised as

$$
\begin{aligned}
\mathbf{u}_{1} & =\mathbf{u}_{\mathbf{d}}-\mathbf{H}_{\mathbf{2}} \mathbf{y}_{\mathbf{2}}, \\
\mathbf{y} & =\mathbf{y}_{\mathbf{1}}, \\
\mathbf{u}_{2 \mathbf{p}} & =\mathbf{F}_{\mathbf{2}} \mathbf{y}_{\mathbf{0}},
\end{aligned}
$$

in which $\mathbf{H}_{2}$ and $\mathbf{F}_{2}$ are the coupling matrices between the two systems, and $\mathbf{y}_{\mathbf{0}}$ represents the plate kinematics, i.e. the $N$ modal displacements, velocities and accelerations of the structure. This can be written according to Eq. (A.1) as

$$
\mathbf{y}_{\mathbf{0}}=\mathbf{C}_{\mathbf{0}} \mathbf{x}_{1}+\mathbf{D}_{\mathbf{0}} \mathbf{u}_{1}
$$

in which

$$
\begin{aligned}
\mathbf{C}_{\mathbf{0}} & =\left[\begin{array}{cc}
\mathbf{I} & \mathbf{0} \\
\mathbf{0} & \mathbf{I} \\
-\mathbf{M}_{p}^{-1} \mathbf{K}_{p} & -\mathbf{M}_{p}^{-1} \mathbf{D}_{p}
\end{array}\right], \\
\mathbf{D}_{\mathbf{0}} & =\left[\begin{array}{c}
\mathbf{D}_{\mathbf{1}} \\
\mathbf{M}_{p}^{-1}
\end{array}\right] .
\end{aligned}
$$

The coupling matrix $\mathbf{H}_{2}$ decomposes the control force from the actuator state-space in the plate modal components, and thus is equal to the $N$ plate eigenvector $\boldsymbol{\phi}_{c}$ at the control position $\left(x_{c}, y_{c}\right)$. Similarly, $\mathbf{F}_{2}$ relates the modal kinematic of the plate to the plate kinematic at the control position, and thus is a block diagonal matrix, in which each block is the transpose eigenvector at the control position $\boldsymbol{\phi}_{c}^{T}$.

The coupling of the two state-space models can be carried out substituting Eq. (A.1) and (A.6a) in Eq. (A.6b), which can be written as

$$
\mathbf{y}=\mathbf{C}_{\mathbf{1}} \mathbf{x}_{\mathbf{1}}+\mathbf{D}_{\mathbf{1}} \mathbf{u}_{\mathbf{d}}-\mathbf{D}_{\mathbf{1}} \mathbf{H}_{\mathbf{2}} \mathbf{y}_{\mathbf{2}} .
$$

Similarly, the output $\mathbf{y}_{\mathbf{2}}$ can be rewritten substituting Eq. (A.6c) in Eq. (A.3), obtaining

$$
\mathbf{y}_{2}=\mathbf{C}_{2} \mathbf{x}_{2}+\mathbf{D}_{2 \mathrm{a}} \mathbf{u}_{\mathbf{a}}-\mathbf{D}_{2 \mathrm{p}} \mathbf{F}_{2} \mathbf{y}_{\mathbf{0}}
$$

Finally, from Eq. (A.6a) and (A.10) in Eq. (A.7), $\mathbf{y}_{\mathbf{0}}$ can be written as

$$
\mathbf{y}_{0}=\mathbf{N}_{0} C_{0} x_{1}-N_{0} D_{0} H_{2} C_{2} x_{2}+N_{0} D_{0} u_{d}-N_{0} D_{0} H_{2} D_{2 a} u_{a}
$$

with

$$
\mathbf{N}_{\mathbf{0}}=\left(\mathbf{I}+\mathbf{D}_{\mathbf{0}} \mathbf{H}_{\mathbf{2}} \mathbf{D}_{\mathbf{2}} \mathbf{F}_{2}\right)^{-1}
$$

Substituting recursively Eq. (A.11) in Eq. (A.9) and (A.10), and then in the state-space models in Eq. (A.1) and (A.3), the state-space matrices introduced in Eq. (A.5) can be expressed as

$$
\begin{aligned}
& A=\left[\begin{array}{cc}
A_{1}-B_{1} H_{2} D_{2 p} F_{2} N_{0} C_{0} & B_{1} H_{2}\left(D_{2 p} F_{2} \mathbf{N}_{0} \mathbf{D}_{0} H_{2} C_{2}-C_{2}\right) \\
B_{2 p} F_{2} \mathbf{N}_{0} C_{0} & A_{2}-B_{2 p} F_{2} \mathbf{N}_{0} \mathbf{D}_{0} H_{2} C_{2}
\end{array}\right], \\
& \mathbf{B}_{\mathrm{d}}=\left[\begin{array}{c}
\mathbf{B}_{1}-\mathbf{B}_{1} \mathbf{H}_{2} \mathbf{D}_{2 \mathrm{p}} \mathbf{F}_{2} \mathbf{N}_{\mathbf{0}} \mathbf{D}_{0} \\
\mathbf{B}_{2 \mathrm{p}} \mathbf{F}_{2} \mathbf{N}_{\mathbf{0}} \mathbf{D}_{\mathbf{0}}
\end{array}\right], \\
& B_{c}=\left[\begin{array}{c}
B_{1} H_{2}\left(D_{2 p} F_{2} N_{0} D_{0} H_{2} D_{2 a}-D_{2 a}\right) \\
B_{2 a}-B_{2 p} F_{2} N_{0} H_{2} D_{2 a}
\end{array}\right] \\
& C=\left[\begin{array}{ll}
C_{1}-D_{1} H_{2} D_{2 p} F_{2} N_{0} C_{0} & D_{1} H_{2}\left(D_{2 p} F_{2} N_{0} D_{0} H_{2} C_{2}-C_{2}\right)
\end{array}\right], \\
& \mathbf{D}_{\mathrm{d}}=\left[\mathrm{D}_{1}-\mathbf{D}_{1} \mathbf{H}_{2} \mathbf{D}_{2 \mathrm{p}} \mathbf{F}_{2} \mathbf{N}_{\mathbf{0}} \mathbf{D}_{0}\right] \text {, } \\
& \mathbf{D}_{c}=\left[\mathbf{D}_{1} \mathbf{H}_{2}\left(\mathbf{D}_{2 \mathrm{p}} \mathbf{F}_{2} \mathbf{N}_{0} \mathbf{D}_{0} \mathbf{H}_{2} \mathbf{D}_{2 \mathrm{a}}-\mathbf{D}_{2 \mathrm{a}}\right)\right] \text {. }
\end{aligned}
$$


If a velocity feedback control is introduced, in which an control signal $\mathbf{u}_{\mathbf{c}}$ is generated, proportional to the plate velocity at the control point, the control law can be written in the state-space as

$$
\mathbf{u}_{\mathbf{c}}=-g \dot{w}_{c}=-g \boldsymbol{\phi}_{c}^{T} \mathbf{y}
$$

Introducing this feedback relation in the second row of Eq. (A.5), the output of the control system can be rewritten as

$$
\mathbf{y}=\mathbf{N C x}+\mathbf{N D}_{\mathbf{d}} \mathbf{u}_{\mathbf{d}}
$$

with

$$
\mathbf{N}=\left(\mathbf{I}+g \boldsymbol{\phi}_{c}^{T} \mathbf{D}_{\mathbf{c}}\right)^{-1} .
$$

The closed-loop state-space model is obtained introducing the feedback law from Eq. (A.14) in the controlled state-space model of Eq. (A.5), as

$$
\left\{\begin{array}{l}
\dot{\mathbf{x}}=\left[\mathbf{A}-\mathbf{B}_{\mathbf{c}} g \boldsymbol{\phi}_{c}^{T} \mathbf{N C}\right] \mathbf{x}+\left[\mathbf{B}_{\mathbf{d}}-\mathbf{B}_{\mathbf{c}} g \boldsymbol{\phi}_{c}^{T} \mathbf{N} \mathbf{D}_{\mathbf{d}}\right] \mathbf{u}_{\mathbf{d}} \\
\mathbf{y}=\mathbf{N C x}+\mathbf{N D}_{\mathbf{d}} \mathbf{u}_{\mathbf{d}}
\end{array} .\right.
$$

The general formulation presented so far can be greatly simplified in this study, in which $\mathbf{D}_{\mathbf{1}}$ is a null matrix. In this case, the state-space model in Eq. (A.17) simplifies to

$$
\left\{\begin{array}{l}
\dot{\mathbf{x}}=\mathbf{A}_{g} \mathbf{x}+\mathbf{B}_{\mathbf{d}} \mathbf{u}_{\mathbf{d}} \\
\mathbf{y}=\mathbf{C x}
\end{array}\right.
$$

in which $\mathbf{A}_{g}=\mathbf{A}-\mathbf{B}_{\mathbf{c}} g \boldsymbol{\phi}_{c}^{T} \mathbf{C}$.

Finally, in order to assess the absorbed power by the actuator from the structure, the velocity at the control position, and the control force have to be written with respect to the state variables. The velocity at the control position $\dot{w}_{c}$ can be written as

$$
\dot{w}_{c}=\boldsymbol{\phi}_{c}^{T} \mathbf{C x}=\mathbf{C}_{v} \mathbf{x} .
$$

The control force is equal to the output of the actuator state-space model in Eq. (A.3), which can be rewritten from Eq. (A.10), with respect to the global state-variables as

$$
\begin{aligned}
& f_{c}=\mathbf{y}_{\mathbf{2}}=\left[\begin{array}{ll}
-\mathbf{D}_{\mathbf{2}} \mathbf{F}_{\mathbf{2}} \mathbf{N}_{\mathbf{0}} \mathbf{C}_{\mathbf{0}} & \mathbf{C}_{2}+\mathbf{D}_{\mathbf{2}} \mathbf{F}_{\mathbf{2}} \mathbf{N}_{\mathbf{0}} \mathbf{D}_{\mathbf{0}} \mathbf{H}_{\mathbf{2}} \mathbf{C}_{2}
\end{array}\right] \mathbf{x}+ \\
& -\mathbf{D}_{2 \mathrm{p}} \mathbf{F}_{2} \mathbf{N}_{0} \mathbf{D}_{0} \mathbf{u}_{\mathrm{d}}+\left[\mathbf{D}_{2 \mathrm{a}}-\mathbf{D}_{2 \mathrm{p}} \mathbf{F}_{2} \mathbf{N}_{0} \mathbf{D}_{0} \mathbf{H}_{2} \mathbf{D}_{2 \mathrm{a}}\right] \mathbf{u}_{\mathrm{c}}
\end{aligned}
$$

If the velocity feedback of Eq. (A.14) is introduced, the control force reduces to

$$
f_{c}=\mathbf{C}_{f} \mathbf{x}+\mathbf{D}_{f} \mathbf{u}_{\mathbf{d}}
$$

with

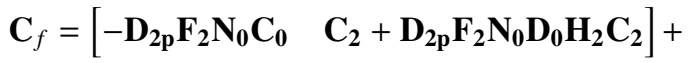

$$
\begin{aligned}
& -\left[\mathbf{D}_{\mathbf{2} \mathbf{a}}-\mathbf{D}_{\mathbf{2} \mathbf{p}} \mathbf{F}_{\mathbf{2}} \mathbf{N}_{\mathbf{0}} \mathbf{D}_{\mathbf{0}} \mathbf{H}_{\mathbf{2}} \mathbf{D}_{\mathbf{2}}\right] g \phi_{c}^{T} \mathbf{N C} \\
& \mathbf{D}_{f}=-\mathbf{D}_{\mathbf{2}} \mathbf{F}_{\mathbf{2}} \mathbf{N}_{\mathbf{0}} \mathbf{D}_{\mathbf{0}}-\left[\mathbf{D}_{\mathbf{2} \mathbf{a}}-\mathbf{D}_{\mathbf{2} \mathbf{p}} \mathbf{F}_{\mathbf{2}} \mathbf{N}_{\mathbf{0}} \mathbf{D}_{\mathbf{0}} \mathbf{H}_{\mathbf{2}} \mathbf{D}_{\mathbf{2}}\right] g \phi_{c}^{T} \mathbf{N D}
\end{aligned}
$$

\section{Appendix B. Comparison between frequency domain and time domain formulation}

The two formulations provided in Section 2 give different results due to different underlying assumptions, which have already been observed in previous work [32], but they have not been completely analysed for small frequency ranges. 
In the frequency domain approach, the integral of the instantaneous kinetic energy with respect to time can be expressed as an integral of the kinetic energy PSD in the frequency domain, using the Parceval Theorem. The integral cannot be written in a closed analytic form, thus numerical integration is carried out as

$$
\bar{K}_{e} \approx \frac{1}{\pi} \int_{0}^{\omega_{\max }} S_{K} d \omega \approx \frac{\Delta \omega}{\pi} \sum_{i=0}^{M-1} S_{K}(i \Delta \omega),
$$

where $\Delta \omega=\omega_{\max } / M$ is the sampling frequency, for which has to be chosen small enough to guarantee the convergence of the summation, choosing a suitable number of frequency points $M$. Nevertheless, the integral of Eq. (B.1) depends on the size of the model, i.e. the number of structural modes $N$ considered, and on the frequency range of integration, defined by $\omega_{\max }$. The upper limit $\omega_{\max }$ has to be chosen large enough to include the low-frequency range, in which the active damping is effective. Secondly, the number of structural modes $N$ considered has to provide a correct estimation of the plate dynamics in the frequency range of integration. This can be obtained including all the structural modes up to $\omega_{\max }$, and then increasing recursively $N$ until the dynamic response of the plate at $\omega_{\max }$ differs for less than $1 \%$ from the previous estimation.

In the time domain approach, the solution of Eq. (9) does not require any integration, but only the computation of the covariance matrix of the steady state response of the system in Eq. (10), and thus it is typically much faster than the frequency domain approach. Moreover, the results obtained depend only on the size of the model $N$, and it corresponds to an integration in the frequency range up to infinity.

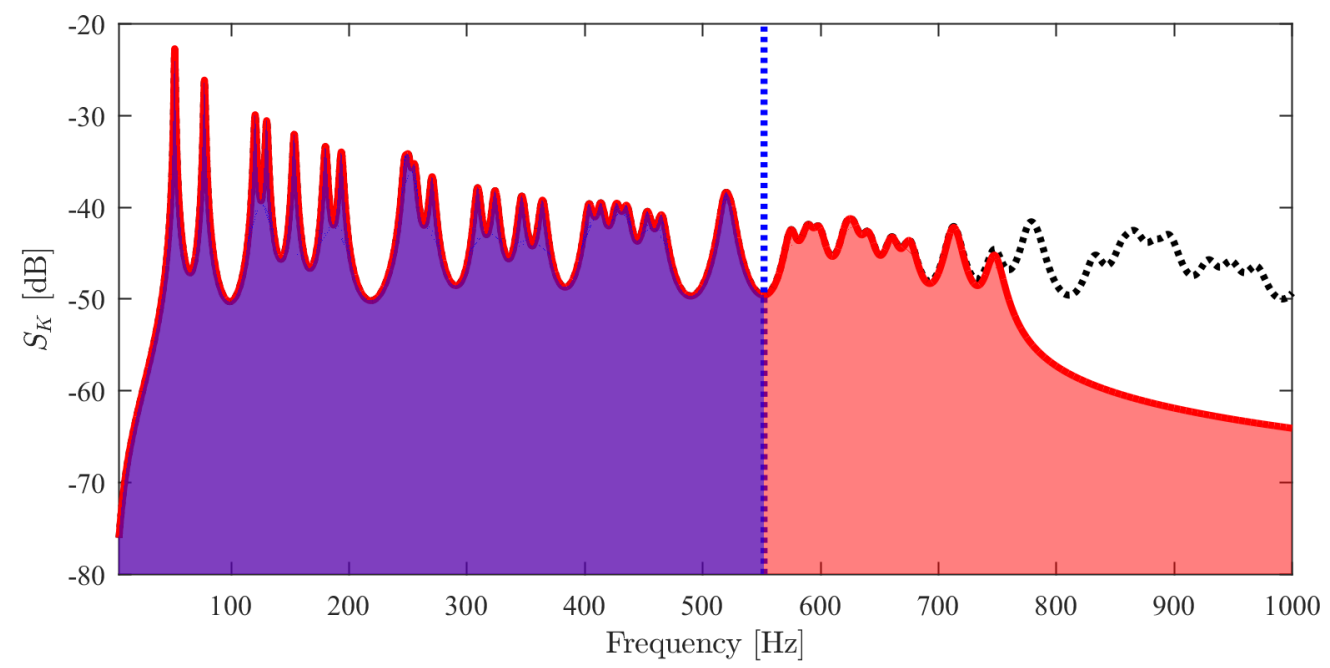

Figure B.17: Kinetic energy PSD of a rectangular plate subjected to rain-on-the-roof excitation, including all the modes up to $1 \mathrm{kHz}$ (dotted black line), and including all the significant modes up to $550 \mathrm{~Hz}$ (solid red line). (For interpretation to the references to colour in this figure, the reader is referred to the web version of this article.)

A comparison between the two formulations is shown in Fig. B.17, in which the dynamic behaviour of plate in investigated up to $550 \mathrm{~Hz}$. The dotted black line shows the 'real' kinetic energy PSD, obtained with a very large model, which includes all the structural modes up to $1 \mathrm{kHz}$. The solid red line shows the model obtained considering only the modes which guarantee an accuracy of the $1 \%$ at $550 \mathrm{~Hz}$. The time-averaged kinetic energy obtained using the frequency domain model is proportional to the area covered by the kinetic energy PSD up to $550 \mathrm{~Hz}$. The timeaveraged kinetic energy obtained using the time domain model, instead, is equivalent to the integral in the frequency domain up to infinity.

Different estimations of the time-averaged kinetic energy are observed depending on the method used. Active damping is typically more effective at low frequencies, in which the dynamic response is mostly determined by the lightly damped resonant peaks. Therefore, it is usually convenient to limit the analysis of the performance only to 
the frequency range in which the active control is effective. This can be easily obtained with the frequency domain approach. Conversely, the time domain approach takes into account all the modes in the model, and thus Eq. (9) has to be modified, in such a way that only the first modes are considered in the time-averaged kinetic energy. This could be done by substituting in the matrix $\mathbf{C}$ in Eq. (9), a diagonal matrix with values of one at the positions corresponding to the first modal velocities of interest [3, 43]. Alternatively, a low-pass filter can be added to the state space model with cut-off frequency which is equal to $\omega_{\max }$. The low-pass filter can be implemented in the model as a second state-space system in series, and the derivation of the state-space matrices is obtained with a procedure similar to the one described in Appendix A. In this paper, both the solutions are implemented, depending on the objective of the analysis.

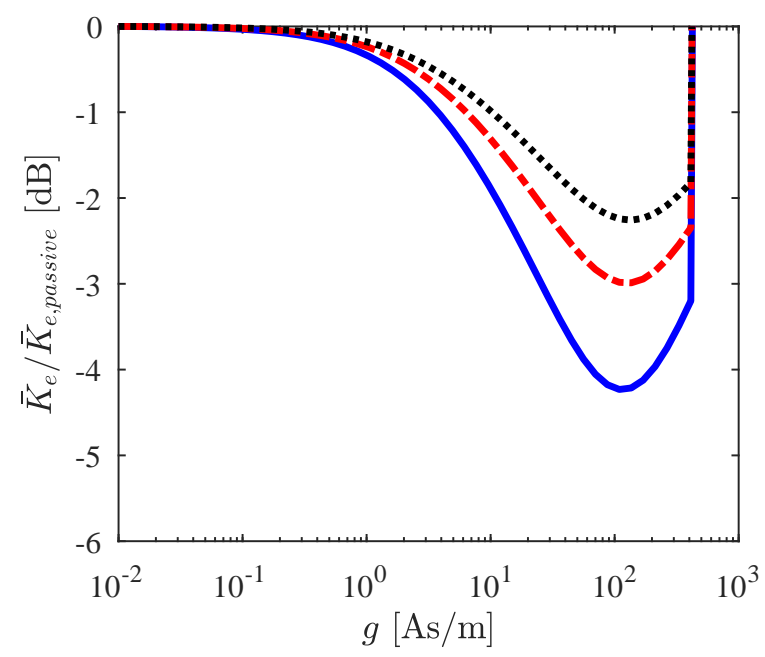

(a)

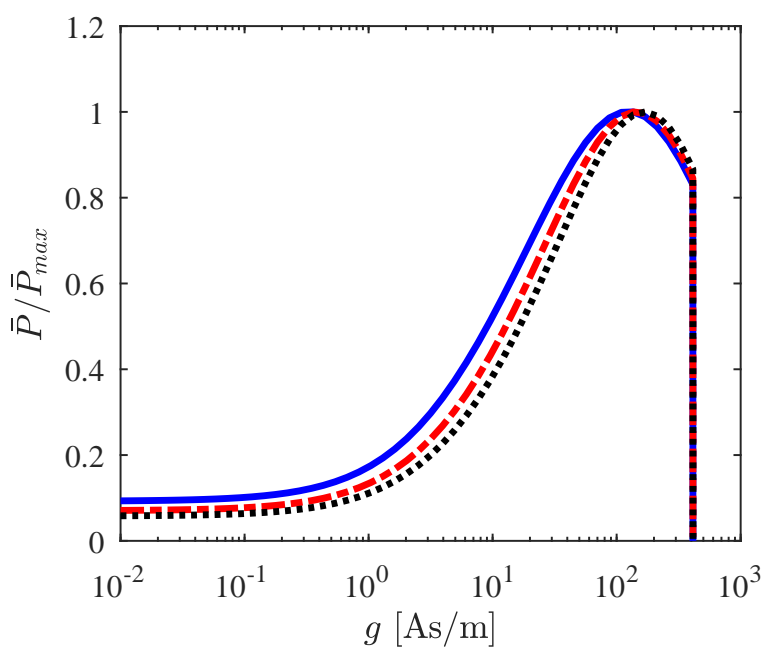

(c)

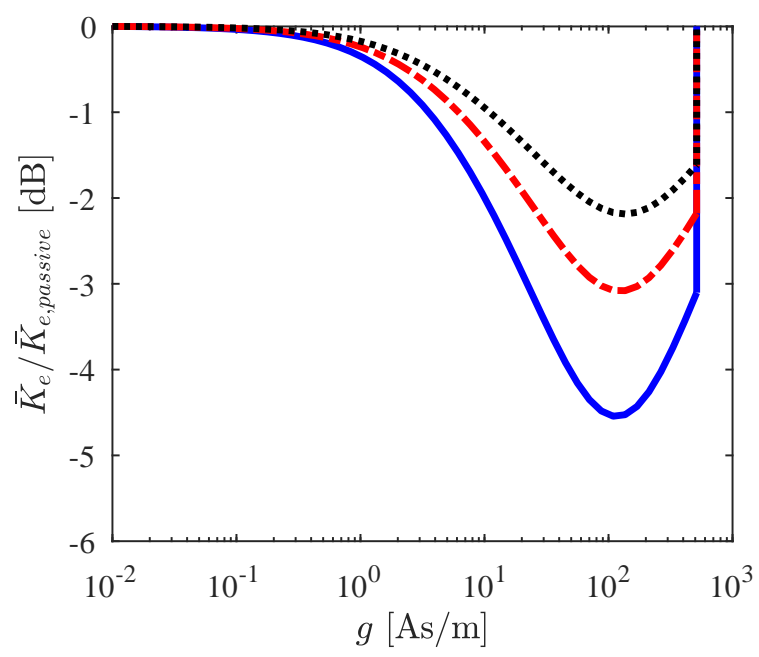

(b)

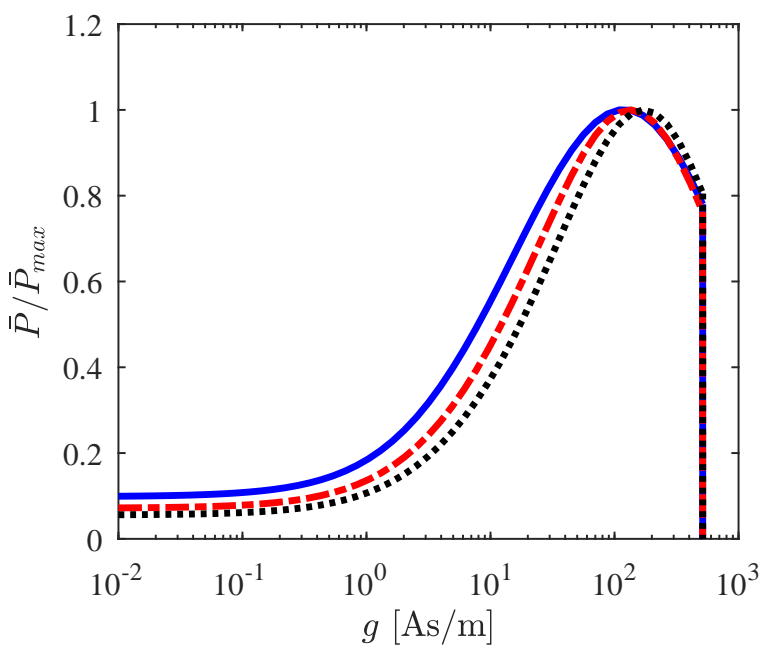

(d)

Figure B.18: $(a, b)$ Time-averaged kinetic energy and $(c, d)$ absorbed power with respect to the feedback gain estimated through (a,c) the time domain and $(b, d)$ the frequency domain approach, for different frequency ranges: up to $250 \mathrm{~Hz}$ (solid blue line), $500 \mathrm{~Hz}$ (dash-dotted red line), and $1 \mathrm{kHz}$ (dotted black line). (For interpretation to the references to colour in this figure, the reader is referred to the web version of this article.)

The results obtained from the two formulations presented in Eq. (9) and Eq. (B.1) for the kinetic energy, and in Eq. (12) and Eq. (6) for the absorbed power, can be compared with respect to the feedback gain $g$, as shown in Fig. B.18. To compare results, a second order Butterworth low-pass filter is included in the state-space formulation. The 
results present similar features to the ideal skyhook damping investigated by Zilletti et al. [1]. except that:

- an upper limit on the feedback gain due to the $180^{\circ}$ phase lag in the force per unit current of the inertial actuator prevents the control system to approach the pinning condition;

- the optimal gain of $109 \mathrm{As} / \mathrm{m}$ does not change significantly with respect to the frequency range of integration, and it is the same for both the formulations;

- the performance of the control system changes with respect to the frequency range of integration, with reductions of vibration up to $-5 \mathrm{~dB}$, but for larger values of $\omega_{\max }$, higher uncontrolled modes are included in the time-average, and thus the estimated effectiveness of the active control reduces;

- the frequency domain formulation provides larger reduction of kinetic energy than the one obtained from the time domain approach, in which, despite the low-pass filter, the higher structural modes are still included in the estimation.

\section{References}

[1] M. Zilletti, S. J. Elliott, P. Gardonio, Self-tuning control systems of decentralised velocity feedback, Journal of Sound and Vibration 329 (14) (2010) 2738-2750.

[2] S. J. Elliott, M. Serrand, P. Gardonio, Feedback stability limits for active isolation systems with reactive and inertial actuators, Journal of Vibration and Acoustics 123 (2) (2001) 250-261.

[3] W. P. Engels, O. N. Baumann, S. J. Elliott, R. Fraanje, Centralized and decentralized control of structural vibration and sound radiation, The journal of the Acoustical Society of America 119 (3) (2006) 1487-1495.

[4] A. Preumont, Vibration control of active structures: an introduction, Vol. 179, Springer Science \& Business Media, 2011.

[5] S. S. Aphale, A. J. Fleming, S. R. Moheimani, Integral resonant control of collocated smart structures, Smart Materials and Structures 16 (2) (2007) 439.

[6] L. Benassi, S. J. Elliott, P. Gardonio, Active vibration isolation using an inertial actuator with local force feedback control, Journal of Sound and Vibration 276 (1) (2004) 157-179. doi:https://doi.org/10.1016/j.jsv.2003.07.019.

[7] I. M. Díaz, P. Reynolds, Acceleration feedback control of human-induced floor vibrations, Engineering Structures 32 (1) (2010) $163-173$

[8] K. Janschek, Mechatronic systems design: methods, models, concepts, Springer Science \& Business Media, 2011.

[9] I. Diaz, P. Reynolds, Robust saturated control of human-induced floor vibrations via a proof-mass actuator, Smart Materials and Structures 18 (12) (2009) 125024

[10] S. J. Elliott, P. Gardonio, T. C. Sors, M. J. Brennan, Active vibroacoustic control with multiple local feedback loops, The Journal of the Acoustical Society of America 111 (2) (2002) 908-915.

[11] P. Gardonio, S. J. Elliott, Smart panels for active structural acoustic control, Smart Materials and structures 13 (6) (2004) 1314.

[12] M. Ray, J. Reddy, Active control of laminated cylindrical shells using piezoelectric fiber reinforced composites, Composites Science and Technology 65 (7-8) (2005) 1226-1236.

[13] F. J. Fahy, P. Gardonio, Sound and structural vibration: radiation, transmission and response, Academic press, 2007.

[14] C. Paulitsch, P. Gardonio, S. J. Elliott, Active vibration damping using an inertial, electrodynamic actuator, Journal of Vibration and Acoustics 129 (1) (2007) 39-47.

[15] M. Zilletti, S. J. Elliott, E. Rustighi, Optimisation of dynamic vibration absorbers to minimise kinetic energy and maximise internal power dissipation, Journal of sound and vibration 331 (18) (2012) 4093-4100. doi:https://doi.org/10.1016/j.jsv.2012.04.023.

[16] J. Rohlfing, P. Gardonio, S. J. Elliott, Base impedance of velocity feedback control units with proof-mass electrodynamic actuators, Journal of Sound and Vibration 330 (20) (2011) 4661 - 4675. doi:https://doi.org/10.1016/j.jsv.2011.04.028.

[17] J. Rohlfing, S. J. Elliott, P. Gardonio, Feedback compensator for control units with proof-mass electrodynamic actuators, Journal of Sound and Vibration 331 (15) (2012) 3437 - 3450. doi:https://doi.org/10.1016/j.jsv.2012.03.010.

[18] S. Camperi, M. Ghandchi-Tehrani, S. J. Elliott, Multichannel decentralised feedback control using inertial actuators, in: Proceedings of the ISMA2016 27th International Conference on Noise and Vibration Engineering, Leuven, Belgium, 2016.

[19] S. Camperi, M. Ghandchi-Tehrani, S. J. Elliott, Experimental maximisation of the power absorbed by an inertial actuator for structural vibration control, in: Proceedings of the ICSV24 24th International Congress on Sound and Vibration, London, United Kingdom, 2017.

[20] S. Camperi, M. Ghandchi-Tehrani, S. J. Elliott, Investigation on the performance of a velocity feedback control unit for structural vibration control: Theory and experiments, in: Proceedings of the IMACXXXVI Conference, Orlando, Florida (USA), 2018.

[21] O. N. Baumann, S. J. Elliott, The stability of decentralized multichannel velocity feedback controllers using inertial actuators, The Journal of the Acoustical Society of America 121 (1) (2007) 188-196.

[22] C. Paulitsch, P. Gardonio, S. J. Elliott, P. Sas, R. Bonnen, Design of a lightweight, electrodynamic, inertial actuator with integrated velocity sensor for active vibration control of a thin lightly-damped panel, in: Proceedings of the ISMA 2004 International Conference on Noise and Vibration Engineering, Leuven, Belgium, 2004.

[23] L. Benassi, S. J. Elliott, Active vibration isolation using an inertial actuator with local displacement feedback control, Journal of Sound and Vibration 278 (4) (2004) 705-724. doi:https://doi.org/10.1016/j.jsv.2003.10.065.

[24] M. Dal Borgo, M. Ghandchi-Tehrani, S. J. Elliott, Nonlinear control and stability analysis of a stroke limited inertial actuator in velocity feedback, in: Proceedings of the ENOC 2017 European Nonlinear Dynamics Conference, Budapest, Hungary, 2017. 
[25] A. Kras, P. Gardonio, Velocity feedback control with a flywheel proof mass actuator, Journal of Sound and Vibration 402 (2017) 31-50. doi:https://doi.org/10.1016/j.jsv.2017.05.001.

[26] I. M. Díaz, E. Pereira, M. J. Hudson, P. Reynolds, Enhancing active vibration control of pedestrian structures using inertial actuators with local feedback control, Engineering Structures 41 (2012) 157-166.

[27] C. Koehne, D. Sachau, K. Renger, Active structural acoustic control in an original a400m aircraft structure, in: Journal of Physics: Conference Series, Vol. 744, IOP Publishing, 2016, p. 012185.

[28] A. Preumont, N. Loix, Active damping of a stiff beam-like structure with acceleration feedback, Experimental Mechanics 34 (1) (1994) 23-26.

[29] T. T. Soong, B. F. Spencer Jr, Supplemental energy dissipation: state-of-the-art and state-of-the-practice, Engineering structures 24 (3) (2002) $243-259$.

[30] K. Tanida, Progress in the application of active vibration control technologies to long-span bridges in japan, Progress in Structural Engineering and Materials 4 (4) (2002) 363-371.

[31] S. J. Elliott, M. Zilletti, P. Gardonio, Self-tuning of local velocity feedback controllers to maximise power absorption, in: Recent Advances Structural Dynamics: Proceedings of the X International Conference, University of Southampton, 2010, p. 12pp.

[32] P. Gardonio, S. Miani, F. Blanchini, D. Casagrande, S. J. Elliott, Plate with decentralised velocity feedback loops: Power absorption and kinetic energy considerations, Journal of sound and vibration 331 (8) (2012) 1722-1741.

[33] M. Zilletti, S. J. Elliott, P. Gardonio, E. Rustighi, Experimental implementation of a self-tuning control system for decentralised velocity feedback, Journal of Sound and Vibration 331 (1) (2012) 1-14

[34] M. Zilletti, P. Gardonio, S. J. Elliott, Optimisation of a velocity feedback controller to minimise kinetic energy and maximise power dissipation, Journal of Sound and Vibration 333 (19) (2014) 4405-4414.

[35] K. Shin, J. Hammond, Fundamentals of signal processing for sound and vibration engineers, John Wiley \& Sons, 2008.

[36] P. Gardonio, M. Zilletti, Integrated tuned vibration absorbers: A theoretical study, The Journal of the Acoustical Society of America 134 (5) (2013) 3631-3644.

[37] S. H. Crandall, Dynamics of mechanical and electromechanical systems, McGraw-Hill, 1968.

[38] S. J. Elliott, M. Zilletti, Scaling of electromagnetic transducers for shunt damping and energy harvesting, Journal of Sound and Vibration 333 (8) (2014) 2185-2195.

[39] L. Cremer, M. Heckl, Structure-borne sound: structural vibrations and sound radiation at audio frequencies, Springer Science \& Business Media, 2013.

[40] S. J. Elliott, Signal processing for active control, Academic press, 2000

[41] L. Meirovitch, Dynamics and control of structures, John Wiley \& Sons, 1990.

[42] E. L. Duke, Combining and connecting linear, multi-input, multi-output subsystem models, Tech. Rep. 19860015695, NASA, United States (1986).

[43] O. N. Baumann, S. J. Elliott, An experiment comparing centralised and decentralised control using inertial actuators, ForumAcusticum 2005. 